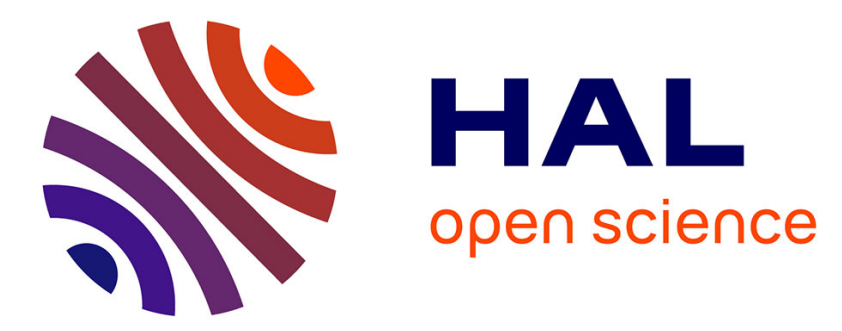

\title{
Critical assessment of a new mathematical model for hysteresis effects on heat and mass transfer in porous building material
}

\author{
Julien Berger, Thomas Busser, Thibaut Colinart, Denys Dutykh
}

\section{To cite this version:}

Julien Berger, Thomas Busser, Thibaut Colinart, Denys Dutykh. Critical assessment of a new mathematical model for hysteresis effects on heat and mass transfer in porous building material. International Journal of Thermal Sciences, 2020, 151, pp.106275. 10.1016/j.ijthermalsci.2020.106275 . hal-02485497

\section{HAL Id: hal-02485497 \\ https://hal.science/hal-02485497}

Submitted on 20 Feb 2020

HAL is a multi-disciplinary open access archive for the deposit and dissemination of scientific research documents, whether they are published or not. The documents may come from teaching and research institutions in France or abroad, or from public or private research centers.
L'archive ouverte pluridisciplinaire HAL, est destinée au dépôt et à la diffusion de documents scientifiques de niveau recherche, publiés ou non, émanant des établissements d'enseignement et de recherche français ou étrangers, des laboratoires publics ou privés.

\section{(1)(1) $\$(0)$}

Distributed under a Creative Commons Attribution - NonCommercial - ShareAlikel 4.0 


\title{
Critical assessment of a new mathematical model for hysteresis effects on heat and mass transfer in porous building material
}

\author{
Julien Berger $^{\mathrm{a} *}$, Thomas Busser ${ }^{\mathrm{a}}$, Thibaut Colinart ${ }^{\mathrm{b}}$, Denys Dutykh ${ }^{\mathrm{c}}$
}

February 20, 2020

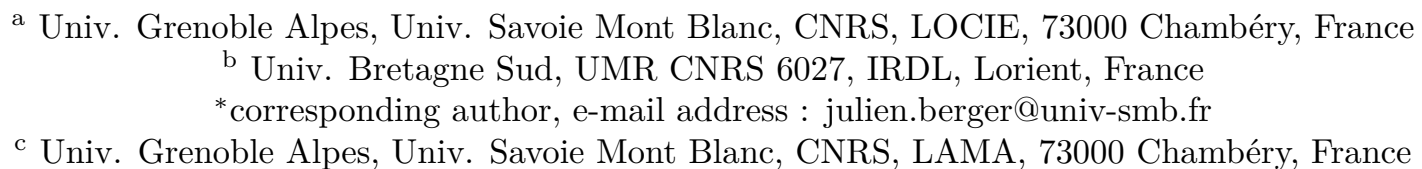

\begin{abstract}
The reliability of mathematical models for heat and mass transfer in building porous material is of capital importance. A reliable model permits to carry predictions of the physical phenomenon with sufficient confidence in the results. Among the physical phenomena, the hysteresis effects on moisture sorption and moisture capacity need to be integrated in the mathematical model of transfer. This article proposes to explore the use of an smooth Bang-Bang model to simulate the hysteresis effects coupled with heat and mass transfer in porous material. This model adds two supplementary differential equations to the two classical ones for heat and mass transfer. The solution of these equations ensures smooth transitions between the main sorption and desorption curves. Two parameters are required to control the speed of transition through the intermediary curves. After the mathematical description of the model, an efficient numerical model is proposed to compute the fields with accuracy and reduced computational efforts. It is based on the DU FORT-FRANKEL scheme for the heat and mass balance equations. For the hysteresis numerical model, an innovative implicit-explicit approach is proposed. Then, the predictions of the numerical model are compared with experimental observations from literature for two case studies. The first one corresponds to a slow cycle of adsorption and desorption while the second is based on a fast cycling case with alternative increase and decrease of moisture content. The comparisons highlight a very satisfactory agreement between the numerical predictions and the observations. In the last Section, the reliability and efficiency of the proposed model is investigated for long term simulation cases. The importance of considering hysteresis effects in the reliability of the predictions are enhanced by comparison with classical approaches from literature.
\end{abstract}

Key words: hysteresis; moisture sorption; porous material; heat and mass transfer; Bang-Bang model; DU FORT-FrANKEL scheme

\section{Introduction}

Within the environmental context, requirements on building energy efficiency become more and more important. Since the latent heat transfer impact strongly the heat losses, several numerical models have been proposed in the literature to predict the physical phenomena occurring through the porous walls. A first overview of these models may be consulted in [1].

It is crucial to have reliable models to predict the physical phenomena with fidelity. Among all the physical phenomena involved in the heat and mass transfer processes through building porous material, the mathematical model to describe the amount of moisture stored in the material is a capital issue. Generally, the moisture content in the material is related to another potential such as relative humidity of capillary pressure. This model is the so-called sorption curve or water retention curve, considered as a material property. Some hysteresis effects occurs on the moisture content in the material and commented in early works of Richards [2, Section Relation between Capillary Potential and Moisture Content]. In other words, the latter strongly depends on the evolution history of the material. The hysteresis processes have significant influence on the precision of the numerical predictions. Coarse modeling of the moisture content in the material lead to inaccurate predictions of heat and mass transfer and their associated consequences.

Various works in the literature highlight these results. In [3-5], it was demonstrated that the simulation of material moisture buffering capacity is inaccurate without hysteresis effects. In [6], the absence of hysteresis 
in the model yield in the prediction of erroneous indoor air comfort. In [7], the prediction of the durability risks of material are more accurate with hysteresis effects. In $[8,9]$, the importance of hysteresis effects on mold growth on wood material is enhanced. Thus, the issue stems from including the hysteresis effects in the mathematical model describing the moisture content in the material.

Several models for hysteresis effects on moisture content have been proposed in the literature. An extended literature review may be consulted in [10]. Among them, one can mention MUALEM's model which is widely used in building physics in [11-13]. The model developed by PEDERSEN [14, 15] is empirical and also widely used thanks to its simplicity. Recently, the so-called gripped box model has been proposed in [16] to improve the accuracy of the predictions of the hysteresis effects and to avoid the so-called pumping numerical errors observed in several models from the literature. If several models have been explored in the literature, it is still an open challenge to propose truly efficient numerical models for hysteresis that can be coupled with simultaneous heat and moisture transfer. The main issue is to reduce the computational efforts without loosing substantially the accuracy while predicting the physical phenomena.

Recently, the so-called Bang-Bang model, inspired by the control literature [17-19], has been introduced to take into account the hysteresis effects on moisture sorption capacity in a model of heat and mass transfer [5]. In [20], the model is coupled with a moisture transport equation to determine the optimal experiment design to estimate the coefficients of the moisture sorption curves. This hysteresis model is based on an additional differential equation enabling smooth transitions between the main adsorption and desorption curves. Results from these works highlight successful use of the Bang-Bang model to take into account the hysteresis effects. To go further, this article intends to explore deeper the fidelity of this approach to represent the hysteresis effects on both moisture content and moisture sorption capacity. It is coupled with a model of heat and mass transfer in porous material. The reliability of the model is investigated by comparing the numerical predictions against experimental observations from the literature. Finally, the efficiency of the numerical model enables to perform simulations over one physical year to investigate the long-term importance of including hysteresis effects to evaluate the thermal loads of a hemp concrete wall submitted to real climate data conditions.

The article is organized as follows. Section 2 presents the mathematical model to represent the physical phenomena of heat and moisture transfer in porous media with hysteresis effects. Then, in Section 3 , an efficient numerical model is proposed to save computational efforts without reducing the accuracy of the solutions. The one-dimensional heat and mass balance equation are solved using the DU FORT-FRANKEL approach while the Bang-Bang model uses an implicit-explicit numerical schemes. The reliability of the model is evaluated in Section 4. Finally, the influence of hysteresis on the prediction of the thermal loads is investigated in Section 5.

\section{Formulation of the mathematical model}

\subsection{Porous material}

The physical phenomena of transfer occurs through the material considered as a porous media [21, 22]. A schematic illustration is given in Figure 1. The media is composed of a solid matrix. The term moisture designates the water vapor and liquid water, both present in the porous. Since the transfer are assumed as one-dimensional, the spatial domain is defined as $x \in[0, L], L[\mathrm{~m}]$ being the thickness of the material.

The physical phenomena are observed for the time interval $t \in[0, \tau]$, where $\tau$ is the final time also called horizon of the simulation. The temperature $T[\mathrm{~K}]$ and relative humidity $\phi[\varnothing]$ in the material are defined as follows:

$$
\begin{aligned}
T:[0, L] \times[0, \tau] & \longrightarrow[273.15,313.15], \\
(x, t) & \longmapsto T(x, t), \\
\phi:[0, L] \times[0, \tau] & \longrightarrow[0,1] \\
(x, t) & \longmapsto \phi(x, t) .
\end{aligned}
$$




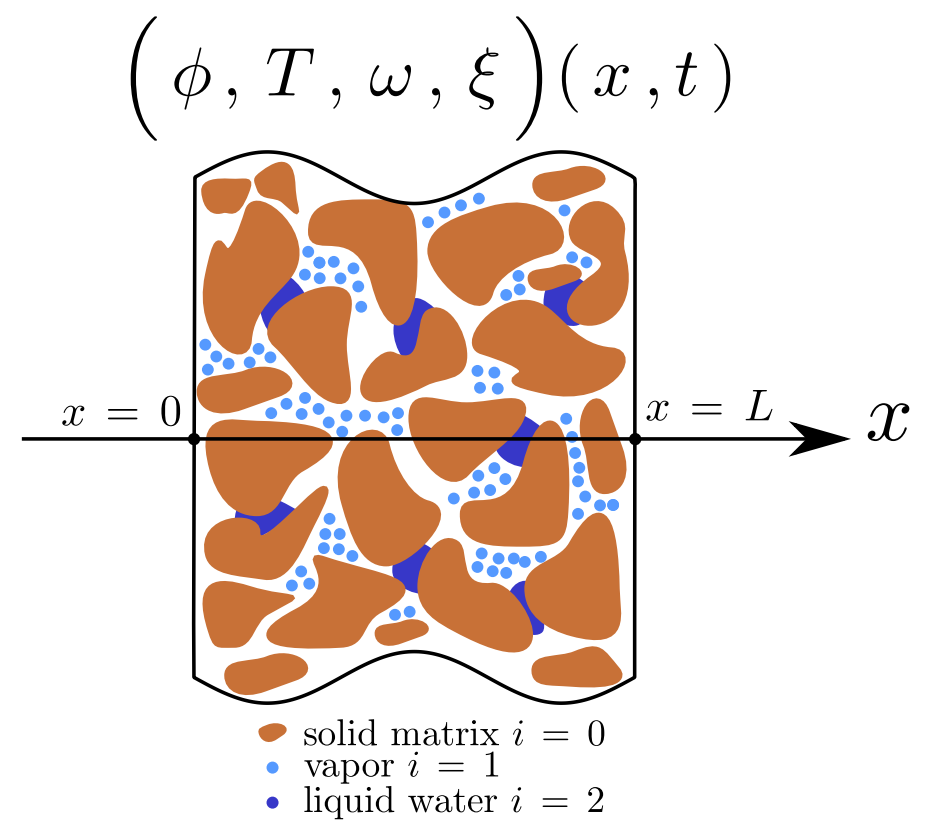

Figure 1. Illustration of a porous material.

The moisture content $\omega[\varnothing]$ corresponds to the amount of water vapor and liquid water in the material. It is defined as:

$$
\begin{aligned}
\omega:[0, L] \times[0, \tau] & \longrightarrow\left[0, \omega_{\mathrm{s}}\right], \\
(x, t) & \longmapsto \omega(x, t),
\end{aligned}
$$

where $\omega_{\mathrm{s}}$ is the maximal moisture content achievable in the material. It is reached at saturated stated, i.e. when the porous of the material are full of water. The constitutive relation between $\phi$ and $\omega$ is provided by the so-called sorption curve which is illustrated in Figure 2. The total moisture in a material is determined by the integral:

$$
\begin{aligned}
\omega_{\text {tot }}:[0, \tau] & \longrightarrow \mathbb{R}_{>0}, \\
t & \longmapsto \int_{0}^{L} \omega(x, t) \mathrm{d} x .
\end{aligned}
$$

Last, the sorption capacity $\xi[\varnothing]$ is characterized by the derivative of the moisture content to the relative humidity:

$$
\begin{aligned}
\xi:[0, L] \times[0, \tau] & \longrightarrow \mathbb{R}_{>0} \\
(x, t) & \longmapsto \frac{\partial \omega}{\partial \phi}
\end{aligned}
$$

The moisture content $\omega$ and the sorption capacity $\xi$ are both subjected to hysteresis effects and will be further described in the following Section 2.3.

\subsection{Heat and moisture balance}

The physical phenomena involve one-dimensional heat and moisture transfer through a porous material. The heat transfer is based on the balance energy and occurs by diffusion and latent mechanisms. The moisture transfer is driven by vapor and liquid diffusions. According to [12, 23], the mathematical model to 
represent the energy and mass conservation in a building porous material can be formulated as follows:

$$
\begin{aligned}
c_{\mathrm{M}} \cdot \frac{\partial \phi}{\partial t} & =\frac{\partial}{\partial x}\left(k_{\mathrm{M}} \cdot \frac{\partial \phi}{\partial x}+k_{\mathrm{VT}} \cdot \frac{\partial T}{\partial x}\right), \\
c_{\mathrm{Q}} \cdot \frac{\partial T}{\partial t} & =\frac{\partial}{\partial x}\left(k_{\mathrm{Q}} \cdot \frac{\partial T}{\partial x}\right)+L_{\mathrm{v}} \cdot \frac{\partial}{\partial x}\left(k_{\mathrm{V}} \cdot \frac{\partial \phi}{\partial x}+k_{\mathrm{VT}} \cdot \frac{\partial T}{\partial x}\right),
\end{aligned}
$$

The storage coefficients $c_{\mathrm{M}}\left[\mathrm{kg} / \mathrm{m}^{3}\right]$ and $c_{\mathrm{Q}}\left[\mathrm{J} /\left(\mathrm{m}^{3} \cdot \mathrm{K}\right)\right]$ are given by:

$$
c_{\mathrm{M}}: \xi \longmapsto \rho_{0} \cdot \xi, \quad c_{\mathrm{Q}}: \omega \longmapsto \rho_{0} \cdot\left(c_{0}+\omega \cdot c_{\ell}\right),
$$

where $\rho_{0}\left[\mathrm{~kg} / \mathrm{m}^{3}\right]$ is the dry specific mass of the material and $c_{0}[\mathrm{~J} /(\mathrm{kg} \cdot \mathrm{K})]$ is the dry specific heat. The moisture transport is driven by vapor and liquid transfer under relative humidity gradient. The total moisture diffusion coefficient $k_{\mathrm{M}}[\mathrm{kg} /(\mathrm{m} \cdot \mathrm{s})]$ incorporates liquid $k_{\mathrm{L}}$ and vapor $k_{\mathrm{V}}$ ones:

$$
\begin{aligned}
& k_{\mathrm{M}}:(T, \omega, \xi) \longmapsto k_{\mathrm{L}}(\omega, \xi)+k_{\mathrm{V}}(T), \\
& k_{\mathrm{L}}:(\omega, \xi) \longmapsto \xi \cdot \exp \left(p_{1}+\frac{\omega_{2}}{\omega}\right), \\
& k_{\mathrm{V}}: T \longmapsto \frac{\kappa_{\mathrm{a}}}{\mu} \cdot P_{\mathrm{sat}}(T),
\end{aligned}
$$

where $\kappa_{\mathrm{a}}$ and $\mu$ are the air and material vapor permeabilities, respectively. Constants $p_{1}$ and $\omega_{2}$ are empirical parameters depending on the material. The saturation pressure $P_{\text {sat }}[\mathrm{Pa}]$ depends on the temperature according to:

$$
P_{\mathrm{sat}}: T \longmapsto P_{\mathrm{sat}}^{\circ} \cdot\left(\frac{T-T_{A}}{T_{B}}\right)^{\alpha}, \quad T \geqslant T_{A},
$$

with $P_{\text {sat }}^{\circ}=997.3 \mathrm{~Pa}, T_{A}=159.5 \mathrm{~K}, T_{B}=120.6 \mathrm{~K}$ and $\alpha=8.275$. The diffusion coefficient $k_{\mathrm{VT}}[\mathrm{kg} /(\mathrm{m} \cdot \mathrm{s} \cdot \mathrm{K})]$ corresponds to the vapor transport under temperature gradient. It is defined by:

$$
k_{\mathrm{VT}}:(\phi, T) \longmapsto \frac{\kappa_{\mathrm{a}}}{\mu} \cdot \phi \cdot \frac{\mathrm{d} P_{\mathrm{sat}}}{\mathrm{d} T} .
$$

The heat transfer coefficient $k_{\mathrm{Q}}[\mathrm{W} /(\mathrm{m} \cdot \mathrm{K})]$ under temperature gradient varies with temperature $T$ and moisture content $\omega$ according to:

$$
k_{\mathrm{Q}}:(T, \omega) \longmapsto k_{\mathrm{Q} 0}+a_{1} \cdot T+b_{1} \cdot \omega .
$$

The latent heat of vaporization $L_{\mathrm{v}}[\mathrm{J} / \mathrm{kg}]$ depends on temperature according to:

$$
L_{\mathrm{v}}: T \longmapsto L_{\mathrm{v}}^{\circ}+\left(c_{\mathrm{v}}-c_{\ell}\right) \cdot\left(T-T_{\mathrm{ref}}\right),
$$

where $c_{\mathrm{v}}[\mathrm{J} /(\mathrm{kg} \cdot \mathrm{K})]$ and $c_{\ell}[\mathrm{J} /(\mathrm{kg} \cdot \mathrm{K})]$ are the specific heat of vapor and liquid water, respectively. The numerical values for the physical constants are $L_{\mathrm{v}}^{\circ}=2.5 \cdot 10^{6} \mathrm{~J} / \mathrm{kg}, c_{\mathrm{v}}=1870 \mathrm{~J} /(\mathrm{kg} \cdot \mathrm{K})$ and $c_{\ell}=4180 \mathrm{~J} /(\mathrm{kg} \cdot \mathrm{K})$. The total heat and moisture fluxes are computed using the following definition:

$$
\begin{aligned}
j_{\mathrm{q}}:(\phi, T, \omega) & \longmapsto k_{\mathrm{Q}}(T, \omega) \cdot \frac{\partial T}{\partial x}+L_{\mathrm{v}}(T) \cdot\left(k_{\mathrm{V}}(T) \cdot \frac{\partial \phi}{\partial x}+k_{\mathrm{VT}}(\phi, T) \cdot \frac{\partial T}{\partial x}\right), \\
j_{\mathrm{m}}:(\phi, T, \omega, \xi) & \longmapsto k_{\mathrm{M}}(T, \omega, \xi) \cdot \frac{\partial \phi}{\partial x}+k_{\mathrm{VT}}(\phi, T) \cdot \frac{\partial T}{\partial x} .
\end{aligned}
$$

They both depend on the space and time coordinates. 


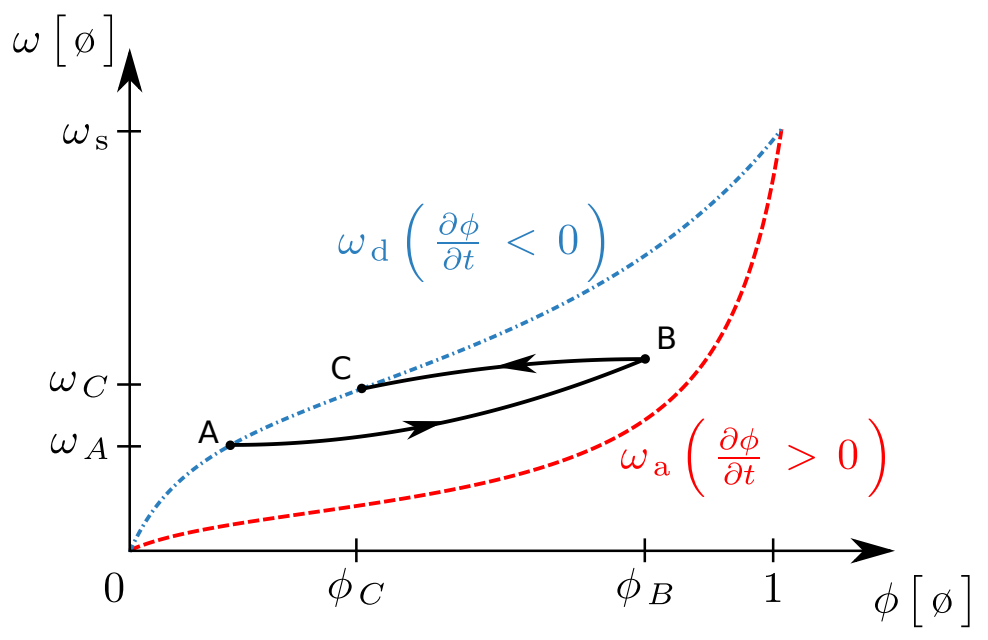

Figure 2. Material moisture content as a function of the relative humidity. The main adsorption $\omega_{\mathrm{a}}$ and desorption $\omega_{\mathrm{d}}$ curves are represented in red (dashed) and blue (dot-dashed) lines, respectively. The black curves illustrate the so-called scanning hysteresis curves in the configuration space $(\omega, \phi)$.

\subsection{Hysteresis effects on water content and sorption capacity: the Bang-Bang Model}

Hysteresis effects are routinely observed on the material moisture content. One may see Figure 2 for an illustration of its concept. In other words, the moisture content depends on the time evolution of the relative humidity. If a completely dry material $\omega=0$ is submitted to an increase of relative humidity, i.e. $\frac{\partial \phi}{\partial t}>0$, the evolution of the moisture content will follow the main adsorption function $\omega_{\mathrm{a}}$. Reciprocally, if a saturated material $\omega=\omega_{\mathrm{s}}$ is dried, i.e. $\frac{\partial \phi}{\partial t}<0$, then the moisture content in the material is given by the main desorption function $\omega_{\mathrm{d}}$, which is different from the main adsorption function. Last, if a material, at the moisture content $\omega=\omega_{A}$, is first wet until a relative humidity $\phi=\phi_{B}$ and then it is dried until $\phi=\phi_{C}<\phi_{B}$, the moisture content evolution will be given by so-called scanning curves between the main adsorption and desorption limits. Since the moisture capacity is defined as the derivative of the moisture content with respect to the relative humidity, it is also subject to hysteresis effects.

To build a model for the prediction of hysteresis effects on the moisture content, we define the main adsorption $\omega_{\mathrm{a}}$ and desorption $\omega_{\mathrm{d}}$ functions:

$$
\begin{array}{rlrl}
\omega_{\mathrm{a}}: \phi \longmapsto \omega_{\mathrm{a}}(\phi), & & \frac{\partial \phi}{\partial t}>0, \\
\omega_{\mathrm{d}}: \phi \longmapsto \omega_{\mathrm{d}}(\phi), & \frac{\partial \phi}{\partial t}<0 .
\end{array}
$$

Both functions $\omega_{\mathrm{a}}$ and $\omega_{\mathrm{d}}$ depend on the relative humidity $\phi$ variable. The former provides the amount of water content when the relative humidity is increasing $\frac{\partial \phi}{\partial t}>0$ and the latter when the relative humidity is decreasing $\frac{\partial \phi}{\partial t}<0$. The adsorption $\Omega_{\mathrm{a}}$ and desorption $\Omega_{\mathrm{d}}$ curves are defined by:

$$
\begin{aligned}
& \Omega_{\mathrm{a}}=\left\{\phi \in[0,1] \mid\left(\phi, \omega_{\mathrm{a}}(\phi)\right)\right\} \subseteq[0,1] \times\left[0, \omega_{\mathrm{s}}\right], \\
& \Omega_{\mathrm{d}}=\left\{\phi \in[0,1] \mid\left(\phi, \omega_{\mathrm{d}}(\phi)\right)\right\} \subseteq[0,1] \times\left[0, \omega_{\mathrm{s}}\right] .
\end{aligned}
$$

The so-called Bang-Bang model originates from control literature theory with an introduction of its basics concepts in [19, Chapter 11]. The original version of the model enables to switch between two states and as far as we are concerned between the main adsorption $\Omega_{\mathrm{a}}$ and desorption $\Omega_{\mathrm{d}}$ curves. The switch is discontinuous between the two curves. In other words, with the original model the moisture capacity only 
takes values of the main sorption or desorption curves $\omega \in \Omega_{\mathrm{a}} \cup \Omega_{\mathrm{d}}$. This approach is not convenient to model the hysteresis in porous material since the moisture sorption values may take intermediary values between the two main sorption $\Omega_{\mathrm{a}}$ and desorption curves $\Omega_{\mathrm{d}}$. Therefore, a smooth version of the BangBang model is introduced to compute the intermediate scanning curves using the main adsorption $\omega_{\mathrm{a}}$ and desorption $\omega_{\mathrm{d}}$ functions:

$$
\frac{\partial \omega}{\partial t}=-s \cdot \beta(s) \cdot\left(\omega-\omega_{\mathrm{d}}\right) \cdot\left(\omega-\omega_{\mathrm{a}}\right),
$$

where the function $s$ is defined as:

$$
s: \phi \longmapsto \operatorname{sign}\left(\frac{\partial \phi}{\partial t}\right)
$$

and the function sign is traditionally given by:

$$
\operatorname{sign}(x)= \begin{cases}1, & x>0 \\ 0, & x=0 \\ -1, & x<0\end{cases}
$$

The function $\beta\left[\mathrm{s}^{-1}\right]$ is specific to the material and depending on the adsorption and desorption processes:

$$
\begin{aligned}
\beta:\{-1,0,1\} & \longrightarrow\left\{\beta_{\mathrm{a}}, \beta_{\mathrm{d}}\right\}, \\
s & \longmapsto \begin{cases}\beta_{\mathrm{a}}, & s \geqslant 0, \\
\beta_{\mathrm{d}}, & s<0,\end{cases}
\end{aligned}
$$

knowing that $\left\{\beta_{\mathrm{a}}, \beta_{\mathrm{d}}\right\} \in \mathbb{R}_{>0}$.

To explain roughly the philosophy of the model, we assume fixed values of $\omega_{\mathrm{a}}$ and $\omega_{\mathrm{d}}$, such as $\omega_{\mathrm{a}}<\omega_{\mathrm{d}}$. The initial value of moisture content at $t=0$ is denoted by $\omega_{0}$. With these assumptions, an analytical solution of Eq. (4) can be computed:

$$
\omega: t \longmapsto \frac{\omega_{\mathrm{a}} \cdot \exp \left(\left(\omega_{\mathrm{d}}-\omega_{\mathrm{a}}\right) \cdot s \cdot \beta(s) \cdot t\right) \cdot\left(\omega_{0}-\omega_{\mathrm{d}}\right)+\omega_{\mathrm{d}} \cdot\left(\omega_{\mathrm{a}}-\omega_{0}\right)}{\exp \left(\left(\omega_{\mathrm{d}}-\omega_{\mathrm{a}}\right) \cdot s \cdot \beta(s) \cdot t\right) \cdot\left(\omega_{0}-\omega_{\mathrm{d}}\right)+\omega_{\mathrm{a}}-\omega_{0}}
$$

and we have the following asymptotic behavior:

$$
\lim _{t \rightarrow \infty} w(t)=\left\{\begin{array}{lll}
\omega_{\mathrm{a}}, & s=1, & \operatorname{sign}\left(\frac{\partial \phi}{\partial t}\right)>0, \\
\omega_{0}, & s=0, & \operatorname{sign}\left(\frac{\partial \phi}{\partial t}\right)=0, \\
\omega_{\mathrm{d}}, & s=-1, & \operatorname{sign}\left(\frac{\partial \phi}{\partial t}\right)<0 .
\end{array}\right.
$$

Thus, when the relative humidity is increasing or decreasing in the material, the solution of Eq. (4) tends to the moisture content value from the main adsorption $\Omega_{\mathrm{a}}$ or desorption $\Omega_{\mathrm{d}}$ curves, respectively. The speed of convergence depends on the parameter $\beta$. In the limits $\beta \rightarrow \infty$ we recover formally the pure Bang-Bang model. For finite values of $\beta$, the transition is smooth.

To obtain the sorption capacity $\xi$, Eq. (4) is differentiated with respect to $\phi$ to obtain the following differential equation:

$$
\frac{\partial \xi}{\partial t}=-s \cdot \beta(s) \cdot\left(\xi-\xi_{\mathrm{d}}\right) \cdot\left(\omega-\omega_{\mathrm{a}}\right)-s \cdot \beta(s) \cdot\left(\omega-\omega_{\mathrm{d}}\right) \cdot\left(\xi-\xi_{\mathrm{a}}\right),
$$


where $\xi_{\mathrm{d}}(-)$ and $\xi_{\mathrm{a}}(-)$ are functions of $\phi$ obtained by differentiating the functions $\omega_{\mathrm{d}}$ and $\omega_{\mathrm{a}}$ relatively to $\phi$. To obtain Eq. (5), it can be remarked that:

$$
\frac{\partial s}{\partial \phi}=\frac{\partial}{\partial \phi}\left(\operatorname{sign}\left(\frac{\partial \phi}{\partial t}\right)\right)=\delta\left(\frac{\partial \phi}{\partial t}\right) \cdot \frac{\partial}{\partial \phi}\left(\frac{\partial \phi}{\partial t}\right)=0,
$$

where $\delta$ is the Dirac function, derivative of the function sign. It explains why the right-hand side of Eq. (5) only has two terms.

\subsection{Boundary and initial conditions}

At the interface between material and air, the moisture flow depends on the surface and ambient fields:

$$
k_{\mathrm{M}} \cdot \frac{\partial \phi}{\partial n}+k_{\mathrm{VT}} \cdot \frac{\partial T}{\partial n}=h_{\mathrm{V}} \cdot\left(\frac{\phi_{\infty} \cdot P_{\mathrm{sat}, \infty}}{T_{\infty}}-\frac{\phi \cdot P_{\mathrm{sat}}}{T}\right), \quad x \in\{0, L\},
$$

where $T_{\infty}, \phi_{\infty}$ and $P_{\text {sat, } \infty}$ are the ambient air temperature, relative humidity and saturation pressure, respectively, $h_{\mathrm{V}}[\mathrm{K} \cdot \mathrm{s} / \mathrm{m}]$ is the surface vapor transfer coefficient. The derivative is defined as $\frac{\partial y}{\partial n}:=\frac{\partial y}{\partial x} \cdot n$ with $n= \pm 1$, depending on the considered boundary. For impermeable boundary condition, the flux is forced to vanish:

$$
k_{\mathrm{M}} \cdot \frac{\partial \phi}{\partial n}+k_{\mathrm{VT}} \cdot \frac{\partial T}{\partial n}=0, \quad x \in\{0, L\} .
$$

The heat flow also depends on the surface and ambient conditions:

$$
\begin{aligned}
& k_{\mathrm{Q}} \cdot \frac{\partial T}{\partial n}+L_{\mathrm{v}} \cdot\left(k_{\mathrm{V}} \cdot \frac{\partial \phi}{\partial n}+k_{\mathrm{VT}} \cdot \frac{\partial T}{\partial n}\right)= \\
& h_{\mathrm{Q}} \cdot\left(T_{\infty}-T\right)+L_{\mathrm{v}} \cdot h_{\mathrm{V}} \cdot\left(\frac{\phi_{\infty} \cdot P_{\mathrm{sat}, \infty}}{T_{\infty}}-\frac{\phi \cdot P_{\mathrm{sat}}}{T}\right), \quad x \in\{0, L\},
\end{aligned}
$$

where $h_{\mathrm{Q}}\left[\mathrm{W} /\left(\mathrm{m}^{2} \cdot \mathrm{K}\right)\right]$ is the surface heat transfer coefficient varying with temperature according to:

$$
h_{\mathrm{Q}}=h_{0}+\frac{1}{2} \cdot \epsilon \cdot \sigma \cdot\left(T_{\infty}+T\right)^{3},
$$

with $\epsilon[\varnothing]$ is the emissivity of the material and $\sigma=5.6 \cdot 10^{-8}\left[\mathrm{~W} /\left(\mathrm{m}^{2} \cdot \mathrm{K}^{4}\right)\right]$ is the STEFAn-BoltzMAnN constant. The relation between the surface heat and vapor transfer coefficients is:

$$
h_{\mathrm{V}}=\nu_{0} \cdot h_{0},
$$

where $\nu_{0} \simeq 1.66 \cdot 10^{-6}$ is a coefficient related to the ratio between the air thermal diffusivity and conductivity. If the temperature is prescribed, then a DIRICHLET type boundary conditions is written:

$$
T=T_{\infty}(t), \quad x \in\{0, L\} .
$$

To obtain a well-posed problem, initial conditions have to be prescribed. At the initial state, the fields may depend on space:

$$
T=T_{0}(x), \quad \phi=\phi_{0}(x), \quad \omega=\omega_{0}(x), \quad \xi=\xi_{0}(x) .
$$

with $T_{0}, \phi_{0}, \omega_{0}$ and $\xi_{0}$ given functions of $x$. To have a well-posed problem, initial and boundary conditions must be compatible. 


\subsection{Dimensionless problem}

As discussed and thoroughly motivated in [24-26], it is of capital importance to obtain a dimensionless problem before elaborating a numerical model. For this, dimensionless fields are defined:

$$
u=\frac{\phi}{\phi^{\circ}}, \quad \quad v=\frac{T}{T^{\circ}}, \quad \quad \mathrm{w}=\frac{\omega}{\omega^{\circ}}, \quad y=\frac{\xi}{\xi^{\circ}},
$$

where the reference parameters, denoted by superscript $(-)^{\circ}$, are chosen according to the magnitude of variations of the fields. The space and time variables are also transformed in dimensionless ones:

$$
t^{\star}=\frac{t}{t^{\circ}}, \quad x^{\star}=\frac{x}{L},
$$

with $L$ being the length of the material and $t^{\circ}$ a reference time chosen in accordance with the time scale of the physical phenomena. The latent heat of vaporization, diffusion and storage coefficients are also scaled considering their respective reference values:

$$
\begin{aligned}
& c_{\mathrm{M}}^{\star}=\frac{c_{\mathrm{M}}}{c_{\mathrm{M}}^{\circ}}, \quad \quad c_{\mathrm{Q}}^{\star}=\frac{c_{\mathrm{Q}}}{c_{\mathrm{Q}}^{\circ}} \\
& k_{\mathrm{M}}^{\star}=\frac{k_{\mathrm{M}}}{k_{\mathrm{M}}^{\circ}}, \\
& k_{\mathrm{V}}^{\star}=\frac{k_{\mathrm{V}}}{k_{\mathrm{V}}^{\circ}}, \\
& k_{\mathrm{VT}}^{\star}=\frac{k_{\mathrm{VT}}}{k_{\mathrm{VT}}^{\circ}}, \\
& r^{\star}=\frac{L_{\mathrm{v}}}{L_{\mathrm{v}}^{\circ}} . \\
& k_{\mathrm{Q}}^{\star}=\frac{k_{\mathrm{Q}}}{k_{\mathrm{Q}}^{\circ}},
\end{aligned}
$$

In this way, dimensionless numbers are enhanced enabling to compare the importance of the physical phenomena at the reference state. Two FouRIER numbers are defined to describe the importance of the total heat and moisture transport relative to the diffusion:

$$
\mathrm{Fo}^{\mathrm{M}}=\frac{k_{\mathrm{M}}^{\circ} \cdot t^{\circ}}{c_{\mathrm{M}}^{\circ} \cdot L}, \quad \quad \mathrm{Fo}^{\mathrm{Q}}=\frac{k_{\mathrm{Q}}^{\circ} \cdot t^{\circ}}{c_{\mathrm{Q}}^{\circ} \cdot L} .
$$

The coefficients $\beta, \gamma$ and $\delta$ measure the importance of coupling effects between heat and moisture transport processes:

$$
\beta=\frac{k_{\mathrm{VT}}^{\circ} \cdot T^{\circ}}{k_{\mathrm{M}}^{\circ} \cdot \phi^{\circ}}, \quad \gamma=L_{\mathrm{v}}^{\circ} \cdot \frac{k_{\mathrm{V}}^{\circ} \cdot \phi^{\circ}}{k_{\mathrm{Q}}^{\circ} \cdot T^{\circ}}, \quad \delta=L_{\mathrm{v}}^{\circ} \cdot \frac{k_{\mathrm{VT}}^{\circ}}{k_{\mathrm{Q}}^{\circ}} .
$$

For the boundary conditions, the BIOT numbers are defined to quantify the transfer by diffusion (of moisture or heat) relative to the transfer between the material and the ambient air:

$$
\mathrm{Bi}_{\mathrm{V}}=\frac{h_{\mathrm{V}} \cdot L}{k_{\mathrm{M}}^{\circ}} \cdot \frac{P_{\mathrm{sat}}^{\circ}}{T^{\circ}} \cdot\left(\frac{T^{\circ}}{T_{B}}\right)^{\alpha}, \quad \mathrm{Bi}_{\mathrm{Q}}=\frac{h_{\mathrm{Q}} \cdot L}{k_{\mathrm{Q}}^{\circ}}, \quad \mathrm{Bi}_{\mathrm{QV}}=L_{\mathrm{v}}^{\circ} \cdot \frac{h_{\mathrm{V}} \cdot L}{k_{\mathrm{Q}}^{\circ}} \cdot \frac{\phi^{\circ} \cdot P_{\mathrm{sat}}^{\circ}}{\left(T^{\circ}\right)^{2}} \cdot\left(\frac{T^{\circ}}{T_{B}}\right)^{\alpha} .
$$

The dimensionless function $\theta$ is also defined as:

$$
\theta: v \longmapsto \frac{1}{v} \cdot\left(v-v_{A}\right)^{\alpha}, \quad v_{A}=\frac{T}{T_{A}} .
$$

For the two differential equations (4) and (5) accounting for hysteresis effects, a scaled function is set as:

$$
\beta^{\star}\left(s^{\star}\right)=\beta\left(s^{\star}\right) \cdot \omega^{\circ} \cdot t^{\circ}, \quad s^{\star}=\operatorname{sign}\left(\frac{\partial u}{\partial t}\right) .
$$

Finally, the dimensionless governing equations to simulate the physical phenomena of heat and moisture transport with hysteresis effects on water sorption can be formulated as a system of four coupled partial 
differential equations:

$$
\begin{aligned}
c_{\mathrm{M}}^{\star} \cdot \frac{\partial u}{\partial t^{\star}} & =\mathrm{FO}^{\mathrm{M}} \cdot\left(\frac{\partial}{\partial x^{\star}}\left(k_{\mathrm{M}}^{\star} \cdot \frac{\partial u}{\partial x^{\star}}\right)+\beta \cdot \frac{\partial}{\partial x^{\star}}\left(k_{\mathrm{VT}}^{\star} \cdot \frac{\partial v}{\partial x^{\star}}\right)\right) \\
c_{\mathrm{Q}}^{\star} \cdot \frac{\partial v}{\partial t^{\star}} & =\mathrm{FO}^{\mathrm{Q}} \cdot\left(\frac{\partial}{\partial x^{\star}}\left(k_{\mathrm{Q}}^{\star} \cdot \frac{\partial v}{\partial x^{\star}}\right)+\gamma \cdot r^{\star} \cdot \frac{\partial}{\partial x^{\star}}\left(k_{\mathrm{V}}^{\star} \cdot \frac{\partial u}{\partial x^{\star}}\right)+\delta \cdot r^{\star} \cdot \frac{\partial}{\partial x^{\star}}\left(k_{\mathrm{VT}}^{\star} \cdot \frac{\partial v}{\partial x^{\star}}\right)\right), \\
\frac{\partial \mathrm{w}}{\partial t^{\star}} & =-s^{\star} \cdot \beta^{\star}\left(s^{\star}\right) \cdot\left(\mathrm{w}-\mathrm{w}_{\mathrm{d}}\right) \cdot\left(\mathrm{w}-\mathrm{w}_{\mathrm{a}}\right) \\
\frac{\partial y}{\partial t^{\star}} & =-s^{\star} \cdot \beta^{\star}\left(s^{\star}\right) \cdot\left(y-y_{\mathrm{d}}\right) \cdot\left(\mathrm{w}-\mathrm{w}_{\mathrm{a}}\right)-s^{\star} \cdot \beta^{\star}\left(s^{\star}\right) \cdot\left(\mathrm{w}-\mathrm{w}_{\mathrm{d}}\right) \cdot\left(y-y_{\mathrm{a}}\right)
\end{aligned}
$$

The couplings between Eqs. (10c) and (10d) with Eqs. (10a) and (10b) occur through the coefficients $c_{\mathrm{M}}^{\star}$, $c_{\mathrm{Q}}^{\star}, k_{\mathrm{Q}}^{\star}$ and $k_{\mathrm{M}}^{\star}$ which depend on functions $\mathrm{w}$ and $y$. Furthermore, the field $u$ impacts Eqs. (10c) and (10d) since functions $\omega_{\mathrm{a}}, \omega_{\mathrm{d}}, y_{\mathrm{a}}$ and $y_{\mathrm{d}}$ depend on $u$. The dimensionless boundary conditions are expressed as:

$$
\begin{gathered}
k_{\mathrm{M}}^{\star} \cdot \frac{\partial u}{\partial n^{\star}}+\beta \cdot k_{\mathrm{VT}}^{\star} \cdot \frac{\partial v}{\partial n^{\star}}=\mathrm{Bi}^{\mathrm{M}} \cdot\left(\theta_{\infty} \cdot u_{\infty}-\theta \cdot u\right), \\
k_{\mathrm{Q}}^{\star} \cdot \frac{\partial v}{\partial n^{\star}}+\gamma \cdot r^{\star} \cdot k_{\mathrm{V}}^{\star} \cdot \frac{\partial u}{\partial n^{\star}}+\delta \cdot r^{\star} \cdot k_{\mathrm{VT}}^{\star} \cdot \frac{\partial v}{\partial n^{\star}}=\mathrm{Bi}^{\mathrm{Q}} \cdot\left(v_{\infty}-v\right)+\mathrm{Bi}^{\mathrm{QV}} \cdot\left(\theta_{\infty} \cdot u_{\infty}-\theta \cdot u\right) .
\end{gathered}
$$

Important non-linearities are introduced in the boundary conditions through the function $\theta$ which depends on the field $v$.

\section{Numerical model}

After defining the mathematical model to represent the physical phenomena, the next step is now to define the numerical model used to perform simulations. The numerical model requires to be efficient by saving computational efforts and providing accurate solutions of the mathematical model.

A uniform discretisation is considered for space and time intervals. The discretisation parameters are denoted using $\Delta t$ for the time and $\Delta x$ for the space. The discrete values of function $u(x, t)$ are denoted by $u_{j}^{n} \stackrel{\text { def }}{=} u\left(x_{j}, t^{n}\right)$ with $j=1, \ldots, N_{x}$ and $n=1, \ldots, N_{t}$. Similarly, we have $v\left(x_{j}, t^{n}\right) \equiv v_{j}^{n}$, $\mathrm{w}\left(x_{j}, t^{n}\right) \equiv \mathrm{w}_{j}^{n}$ and $y\left(x_{j}, t^{n}\right) \equiv y_{j}^{n}$. For the sake of clarity, the numerical method is described using a simpler notation by removing the subscript $\star$.

\subsection{Heat and mass balance equations}

To build an efficient numerical model for the heat and mass balance equations (10a) and (10b), the DU FORT-FrANKEL scheme is used. It provides an explicit numerical scheme with an increased stability domain. Interested readers may consult [27] for the original work, [28, 29] for the results on the stability analysis and $[29,30]$ for further details and its applications for heat and moisture transfer in building porous materials. Since a complete description is provided in [30] for an analogous system of coupled diffusion equations, the whole demonstration of the scheme is not detailed here. At the time step $t^{n}$, from Eq. (10a) the computation of the dimensionless relative humidity $u_{j}^{n+1}$ is obtained by the following fully discrete dynamical system:

$$
\begin{array}{r}
u_{j}^{n+1}=\frac{1}{1+\lambda_{3}} \cdot\left(\lambda_{1} \cdot u_{j+1}^{n}+\lambda_{2} \cdot u_{j-1}^{n}+\left(1-\lambda_{3}\right) \cdot u_{j}^{n-1}\right. \\
\left.+\nu_{1} \cdot v_{j+1}^{n}+\nu_{2} \cdot v_{j-1}^{n}-\nu_{3} \cdot v_{j}^{n}\right),
\end{array}
$$


with the coefficients $\left\{\lambda_{i}\right\}_{i=1}^{3}$ and $\left\{\nu_{i}\right\}_{i=1}^{3}$ defined as:

$$
\begin{aligned}
& \lambda_{1} \stackrel{\text { def }}{=} 2 \cdot \mathrm{Fo}^{\mathrm{M}} \cdot \frac{\Delta t}{\Delta x^{2}} \cdot \frac{k_{\mathrm{M}, j+\frac{1}{2}}^{n}}{y^{n}}, \quad \lambda_{2} \stackrel{\text { def }}{=} 2 \cdot \mathrm{Fo}^{\mathrm{M}} \cdot \frac{\Delta t}{\Delta x^{2}} \cdot \frac{k_{\mathrm{M}, j-\frac{1}{2}}^{n}}{y^{n}}, \quad \lambda_{3} \stackrel{\text { def }}{=} \frac{1}{2} \cdot\left(\lambda_{1}+\lambda_{2}\right), \\
& \nu_{1} \stackrel{\text { def }}{=} 2 \cdot \beta \cdot \mathrm{FO}^{\mathrm{M}} \cdot \frac{\Delta t}{\Delta x^{2}} \cdot \frac{k_{\mathrm{VT}, j+\frac{1}{2}}^{n}}{y^{n}}, \quad \nu_{2} \stackrel{\text { def }}{=} 2 \cdot \beta \cdot \mathrm{Fo}^{\mathrm{M}} \cdot \frac{\Delta t}{\Delta x^{2}} \cdot \frac{k_{\mathrm{VT}, j-\frac{1}{2}}^{n}}{y^{n}}, \quad \nu_{3} \stackrel{\text { def }}{=} \nu_{1}+\nu_{2} .
\end{aligned}
$$

These coefficients depend also on $j$ and $n$. Then, from Eq. (10b), the dimensionless temperature $v_{j}^{n+1}$ is computed with:

$$
\begin{array}{r}
v_{j}^{n+1}=\frac{1}{1+\mu_{3}} \cdot\left(\mu_{1} \cdot v_{j+1}^{n}+\mu_{2} \cdot v_{j-1}^{n}+\left(1-\mu_{3}\right) \cdot v_{j}^{n-1}\right. \\
\left.\quad+\rho_{1} \cdot u_{j+1}^{n}+\rho_{2} \cdot u_{j-1}^{n}-\frac{1}{2} \rho_{3} \cdot\left(u_{j}^{n}+u_{j}^{n+1}\right)\right),
\end{array}
$$

and the following coefficients $\mu$ and $\rho$ :

$$
\begin{array}{ll}
\mu_{1} \stackrel{\text { def }}{=} 2 \cdot \mathrm{FO}^{\mathrm{Q}} \cdot \frac{\Delta t}{\Delta x^{2}} \cdot \frac{1}{c_{\mathrm{Q}}^{n}} \cdot\left(k_{\mathrm{Q}, j+\frac{1}{2}}^{n}+\delta \cdot r^{n} \cdot k_{\mathrm{VT}, j+\frac{1}{2}}^{n}\right), & \mu_{3} \stackrel{\text { def }}{=} \frac{1}{2} \cdot\left(\mu_{1}+\mu_{2}\right), \\
\mu_{2} \stackrel{\text { def }}{=} 2 \cdot \mathrm{FO}^{\mathrm{Q}} \cdot \frac{\Delta t}{\Delta x^{2}} \cdot \frac{1}{c_{\mathrm{Q}}^{n}} \cdot\left(k_{\mathrm{Q}, j-\frac{1}{2}}^{n}+\delta \cdot r^{n} \cdot k_{\mathrm{VT}, j-\frac{1}{2}}^{n}\right), & \rho_{3} \stackrel{\text { def }}{=} \rho_{1}+\rho_{2}, \\
\rho_{1} \stackrel{\text { def }}{=} 2 \cdot \gamma \cdot r^{n} \cdot \mathrm{Fo}^{\mathrm{Q}} \cdot \frac{\Delta t}{\Delta x^{2}} \cdot \frac{k_{\mathrm{V}, j+\frac{1}{2}}^{n}}{c_{\mathrm{Q}}^{n}}, & \rho_{2} \stackrel{\text { def }}{=} 2 \cdot \gamma \cdot r^{n} \cdot \mathrm{FO}^{\mathrm{Q}} \cdot \frac{\Delta t}{\Delta x^{2}} \cdot \frac{k_{\mathrm{V}, j-\frac{1}{2}}^{n}}{c_{\mathrm{Q}}^{n}} .
\end{array}
$$

The coefficients $k_{\mathrm{M}}, k_{\mathrm{VT}}, k_{\mathrm{Q}}$ and $k_{\mathrm{V}}$, which depend on the fields $u, v$, w and/or $y$, are evaluated using the following interpolation:

$$
\begin{aligned}
k_{j+\frac{1}{2}}\left(u_{j}, v_{j}, \mathrm{w}_{j}, y_{j}\right) & \stackrel{\text { def }}{=} \\
& k\left(\frac{1}{2} \cdot\left(u_{j}+u_{j+1}\right), \frac{1}{2} \cdot\left(v_{j}+v_{j+1}\right), \frac{1}{2} \cdot\left(\mathrm{w}_{j}+\mathrm{w}_{j+1}\right), \frac{1}{2} \cdot\left(y_{j}+y_{j+1}\right)\right) .
\end{aligned}
$$

It is also recalled that the boundary conditions must be discretised using second order accurate in space to maintain the accuracy uniformly in space properties of the DU FORT-FrANKEL scheme.

\subsection{Hysteresis equations}

An IMplicit-EXplicit (IMEX) method is used to approach the solutions of Eqs. (10c) and (10d). The functions $\mathrm{w}$ and $y$ depend on $t$ and $u$. Thus, the numerical scheme is applied uniformly for all $x_{j}, j \in$ $\left\{1, \ldots, N_{x}\right\}$. However, Eqs. (10c) and (10d) do not involve any spatial derivatives. So, for the seek of clarity and without loss of generality, the subscript $(-)_{j}$ is omitted in the description of the numerical scheme. It is first outlined for Eq. (10c). When the material undergoes an adsorption process $\frac{\partial u}{\partial t}>0$ (or $s=1)$, the numerical scheme is written as:

$$
\frac{1}{\Delta t} \cdot\left(\mathrm{w}^{n+1}-\mathrm{w}^{n}\right)=-s \cdot \beta(s) \cdot\left(\mathrm{w}^{n+1}-\mathrm{w}_{\mathrm{a}}\right) \cdot\left(\mathrm{w}^{n}-\mathrm{w}_{\mathrm{d}}\right),
$$

which gives the following expression for the computation of $w^{n+1}$ :

$$
\mathrm{w}^{n+1}=\frac{\left(\mathrm{w}^{n}+\Delta t \cdot s \cdot \beta(s) \cdot\left(\mathrm{w}^{n}-\mathrm{w}_{\mathrm{d}}\right) \cdot \mathrm{w}_{\mathrm{a}}\right)}{\left(1+\Delta t \cdot s \cdot \beta(s) \cdot\left(\mathrm{w}^{n}-\mathrm{w}_{\mathrm{d}}\right)\right)} .
$$


Similarly, when the material undergoes a desorption process $\frac{\partial u}{\partial t}<0$ (or $s=-1$ ), the IMEX scheme yields:

$$
\frac{1}{\Delta t} \cdot\left(\mathrm{w}^{n+1}-\mathrm{w}^{n}\right)=-s \cdot \beta(s) \cdot\left(\mathrm{w}^{n}-\mathrm{w}_{\mathrm{a}}\right) \cdot\left(\mathrm{w}^{n+1}-\mathrm{w}_{\mathrm{d}}\right),
$$

which leads to:

$$
\mathrm{w}^{n+1}=\frac{\left(\mathrm{w}^{n}+\Delta t \cdot s \cdot \beta(s) \cdot\left(\mathrm{w}^{n}-\mathrm{w}_{\mathrm{a}}\right) \cdot \mathrm{w}_{\mathrm{d}}\right)}{\left(1+\Delta t \cdot s \cdot \beta(s) \cdot\left(\mathrm{w}^{n}-\mathrm{w}_{\mathrm{a}}\right)\right)}
$$

Finally, the computation of $w^{n+1}$ can be expressed by combining the two equations (11) and (12):

$$
\begin{aligned}
\mathrm{w}^{n+1}= & \frac{1}{2} \cdot(1+s) \cdot \frac{\left(\mathrm{w}^{n}+\Delta t \cdot s \cdot \beta(s) \cdot\left(\mathrm{w}^{n}-\mathrm{w}_{\mathrm{d}}\right) \cdot \mathrm{w}_{\mathrm{a}}\right)}{\left(1+\Delta t \cdot s \cdot \beta(s) \cdot\left(\mathrm{w}^{n}-\mathrm{w}_{\mathrm{d}}\right)\right)} \\
& +\frac{1}{2} \cdot(1-s) \cdot \frac{\left(\mathrm{w}^{n}+\Delta t \cdot s \cdot \beta(s) \cdot\left(\mathrm{w}^{n}-\mathrm{w}_{\mathrm{a}}\right) \cdot \mathrm{w}_{\mathrm{d}}\right)}{\left(1+\Delta t \cdot s \cdot \beta(s) \cdot\left(\mathrm{w}^{n}-\mathrm{w}_{\mathrm{a}}\right)\right)} .
\end{aligned}
$$

The extension of the IMEX approach to Eq. (10d) is analogous. Under adsorption process $\frac{\partial u}{\partial t}>0$ (or $s=1$ ), we have:

$$
\begin{aligned}
\frac{1}{\Delta t} \cdot\left(y^{n+1}-y^{n}\right)= & -s \cdot \beta(s) \cdot\left(y^{n+1}-y_{\mathrm{a}}\right) \cdot\left(\mathrm{w}^{n+1}-\mathrm{w}_{\mathrm{d}}\right) \\
& -s \cdot \beta(s) \cdot\left(\mathrm{w}^{n+1}-\mathrm{w}_{\mathrm{a}}\right) \cdot\left(y^{n}-y_{\mathrm{d}}\right)
\end{aligned}
$$

Similarly, under a desorption process $\frac{\partial u}{\partial t}<0$ (or $s=-1$ ), we obtain:

$$
\begin{aligned}
\frac{1}{\Delta t} \cdot\left(y^{n+1}-y^{n}\right)= & -s \cdot \beta(s) \cdot\left(y^{n}-y_{\mathrm{a}}\right) \cdot\left(\mathrm{w}^{n+1}-\mathrm{w}_{\mathrm{d}}\right) \\
& -s \cdot \beta(s) \cdot\left(\mathrm{w}^{n+1}-\mathrm{w}_{\mathrm{a}}\right) \cdot\left(y^{n+1}-y_{\mathrm{d}}\right),
\end{aligned}
$$


which yields the following expression for $y^{n+1}$ :

$$
\begin{aligned}
y^{n+1}= & \frac{1}{2}(1+s) \times \\
& \frac{y^{n}+\Delta t \cdot s \cdot \beta(s) \cdot\left(\mathrm{w}^{n+1}-\mathrm{w}_{\mathrm{d}}\right) \cdot y_{\mathrm{a}}-s \cdot \beta(s) \cdot\left(y^{n}-y_{\mathrm{d}}\right) \cdot\left(\mathrm{w}^{n+1}-\mathrm{w}_{\mathrm{a}}\right)}{1+\Delta t \cdot s \cdot \beta(s) \cdot\left(\mathrm{w}^{n+1}-\mathrm{w}_{\mathrm{d}}\right)} \\
+ & \frac{1}{2}(1-s) \times \\
& \frac{y^{n}+\Delta t \cdot s \cdot \beta(s) \cdot\left(\mathrm{w}^{n+1}-\mathrm{w}_{\mathrm{a}}\right) \cdot y_{\mathrm{d}}-s \cdot \beta(s) \cdot\left(y^{n}-y_{\mathrm{a}}\right) \cdot\left(\mathrm{w}^{n+1}-\mathrm{w}_{\mathrm{d}}\right)}{1+\Delta t \cdot s \cdot \beta(s) \cdot\left(\mathrm{w}^{n+1}-\mathrm{w}_{\mathrm{a}}\right)} .
\end{aligned}
$$

This IMEX approach enables to use the advantages of an fully implicit approach in terms of stability and consistency, but to avoid in the same time the sub-iterations at each time iteration due to the non-linearities. It saves important computational efforts.

\subsection{Efficiency of the numerical model}

The efficiency of a numerical model can be evaluated according to several criteria. First, using a reference solution $u_{\text {ref }}(x, t)$ obtained by measurements, the error of the computed solution $u(x, t)$ can be evaluated:

$$
\varepsilon_{2}(x) \stackrel{\text { def }}{=} \sqrt{\frac{1}{N_{t}} \cdot \sum_{n=1}^{N_{t}}\left(u\left(x, t^{n}\right)-u_{\text {ref }}\left(x, t^{n}\right)\right)^{2}},
$$

where $N_{t}$ is the number of temporal steps where the discrete solution is computed. Similarly, the error can be computed according to time $t$ :

$$
\varepsilon_{2}(t) \stackrel{\text { def }}{=} \sqrt{\frac{1}{N_{x}} \cdot \sum_{j=1}^{N_{x}}\left(u\left(x_{j}, t\right)-u_{\mathrm{ref}}\left(x_{j}, t\right)\right)^{2}},
$$

where $N_{x}$ is the number of spacial nodes where the discrete solution is computed. The simple error between the experimental observations and the numerical predictions $\Delta u$ is defined by:

$$
\Delta u(x, t) \stackrel{\text { def }}{=} u(x, t)-u_{\text {ref }}(x, t) .
$$

The second criterion is the computational (CPU) time required by the numerical model to compute the physical solution. Particularly, the ratio $R_{\mathrm{CPU}}[\mathrm{min} /$ day $]$ between the computational time (measured in minutes) and the time horizon of the simulation (defined in days) is investigated. It is measured using Matlab ${ }^{\mathrm{TM}}$ environment on a computer using Intel i7 CPU and $32 \mathrm{~GB}$ of RAM.

\section{Comparison of numerical predictions with experimental observations}

\subsection{Description of the case}

To evaluate the reliability of the numerical prediction with the proposed mathematical model, experimental results from [12] are used. Samples of sprayed hemp concrete are submitted to steps of relative humidity as illustrated in Figure 3(a). The length of a sample is $L=10 \mathrm{~cm}$. The transfer is assumed to be one-dimensional along axis $x$. Two experimental set-ups are carried out. First, the slow cycling test submit the material to an increase and then a decrease of ambient relative humidity $\phi_{\infty}(t)$. The second case considers fast cycles of increase and decrease of relative humidity. In both cases, the samples weight 


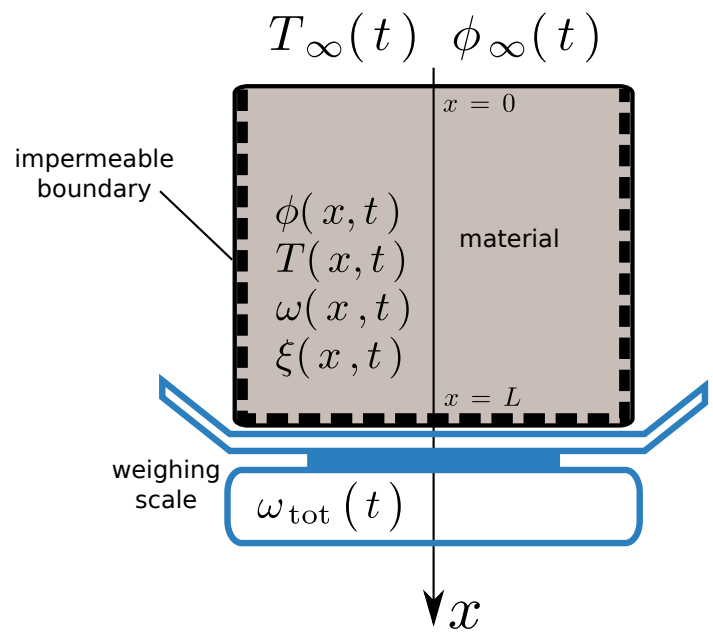

(a)

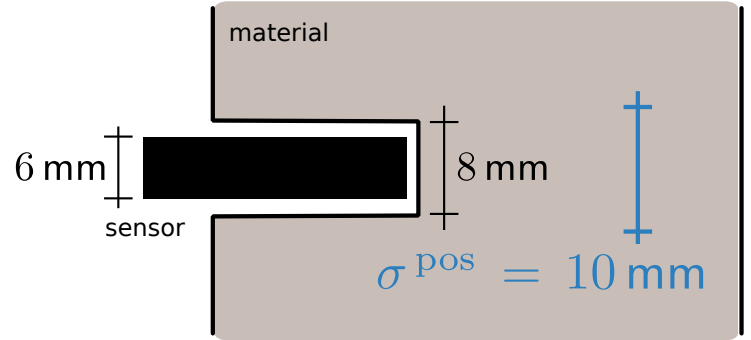

(b)

Figure 3. Illustration of the case study (a) and detail on the insertion of temperature and relative humidity sensors inside the material (b).

is measured with a time interval of $5 \mathrm{~min}$. Additional sensors are set inside the material to monitor the temperature and relative humidity. At the top of the sample $x=0$, RoBIn type boundary conditions are assumed for heat and vapor flows according to Eqs. (6) and (8). At the bottom of the sample $x=L$, the temperature is prescribed using measurements. Thus, a DIRICHLET boundary condition is applied using Eq. (9). This boundary is supposed to be impermeable to water vapor and Eq. (7) is used. Interested readers are invited to consult [12] for further details on the experimental facility and its design.

From the experimental design, two types of experimental observations are obtained. On one hand, there are global measurements of samples weight with respect to time. The uncertainty measurement of the weighing scale is $\sigma_{\omega}^{\text {meas }}=0.01 \mathrm{~g}$. To our best knowledge, we believe that no additional uncertainties may influence the weight of the sample. On the other hand, local sensors provide measurements of temperature and relative humidity in one location $x=x_{0}$ in the material. The total uncertainty of these local measurements depends on the sensor accuracy as well as its location uncertainty in the material. Thus, the spread of uncertainties requires to be evaluated using the following formula [31]:

$$
\sigma_{T}^{2}=\left(\sigma_{T}^{\text {meas }}\right)^{2}+\left(\sigma_{T}^{\text {pos }}\right)^{2}, \quad \sigma_{\phi}^{2}=\left(\sigma_{\phi}^{\text {meas }}\right)^{2}+\left(\sigma_{\phi}^{\text {pos }}\right)^{2},
$$

where $\sigma_{T}$ and $\sigma_{\phi}$ are the total uncertainties on the measurement of temperature and relative humidity, respectively. The quantities $\sigma_{T}^{\text {meas }}$ and $\sigma_{\phi}^{\text {meas }}$ are the sensor measurement uncertainties, provided directly by the supplier. For these Sensirion ${ }^{\mathrm{TM}}$ SHT 75 and $\mathrm{K}$ thermocouples sensors, these quantities are equal to $\sigma_{T}^{\text {meas }}=0.5{ }^{\circ} \mathrm{C}$ and $\sigma_{\phi}^{\text {meas }}=0.02$ for temperature and relative humidity, respectively. The quantities $\sigma_{T}^{\text {pos }}$ and $\sigma_{\phi}^{\text {pos }}$ are the sensor location uncertainties for the temperature and relative humidity sensors. They are computed by:

$$
\sigma_{T}^{\text {pos }}=\left.\frac{\partial T}{\partial x}\right|_{x=x_{0}} \sigma^{\text {pos }}, \quad \sigma_{\phi}^{\text {pos }}=\left.\frac{\partial \phi}{\partial x}\right|_{x=x_{0}} \sigma^{\text {pos }},
$$

where $\sigma^{\text {pos }}$ is the uncertainty on the position of the sensor. According to the experimental design, illustrated in Figure 3(b), the sensor is introduced inside the material by realizing a hole of $8 \mathrm{~mm}$. Thus, with the uncertainty of the drilling, the uncertainty on the position is evaluated to $\sigma^{\text {pos }}=1 \mathrm{~cm}$. The quantities $\left.\frac{\partial T}{\partial x}\right|_{x=x_{0}}$ and $\left.\frac{\partial \phi}{\partial x}\right|_{x=x_{0}}$ are computed using the solution of the numerical model.

\subsection{Material properties}

The material properties of hemp concrete were evaluated previously the experimental measurements. The specific dry mass has been evaluated in [32-35] and equals $\rho_{0}=454 \mathrm{~kg} / \mathrm{m}^{3}$. Using calorimetric 
measurement [12], the dry specific heat is $c_{0}=1000 \mathrm{~J} /(\mathrm{kg} \cdot \mathrm{K})$. According to [36], the thermal conductivity depends on temperature and moisture content as stated in Eq. (3) with the numerical values of the parameters $k_{\mathrm{Q} 0}=8.18 \cdot 10^{-2} \mathrm{~W} /(\mathrm{m} \cdot \mathrm{K}), a_{1}=2.76 \cdot 10^{-4} \mathrm{~W} /\left(\mathrm{m} \cdot \mathrm{K}^{2}\right)$ and $b_{1}=2.4 \cdot 10^{-3} \mathrm{~W} /(\mathrm{m} \cdot \mathrm{K})$. The liquid and vapor diffusion coefficient are defined according to Eq. (2) with the following parameters $[12,37] p_{1}=-13.5, \omega_{2}=-0.12, \kappa_{a}=2 \cdot 10^{-10} \mathrm{~kg} /(\mathrm{m} \cdot \mathrm{s} \cdot \mathrm{Pa})$ and $\mu=5$. The main sorption $\omega_{\mathrm{a}}(\phi)$ and desorption $\omega_{\mathrm{d}}(\phi)$ equations are defined using GuGGenheim-Anderson-De Boer (GAB) model:

$$
\omega_{\{\mathrm{d}, \mathrm{a}\}}(\phi)=\frac{\omega_{m,\{\mathrm{~d}, \mathrm{a}\}} \cdot K_{\{\mathrm{d}, \mathrm{a}\}} \cdot C_{\{\mathrm{d}, \mathrm{a}\}}}{\left(1-K_{\{\mathrm{d}, \mathrm{a}\}} \cdot \phi\right) \cdot\left(1+K_{\{\mathrm{d}, \mathrm{a}\}} \cdot\left(C_{\{\mathrm{d}, \mathrm{a}\}}-1\right) \cdot \phi\right)} \phi,
$$

with the parameters determined in [12] and set to $\omega_{m, \mathrm{a}}=0.02, K_{\mathrm{a}}=0.86$ and $C_{\mathrm{a}}=7$ for the main adsorption equation and $\omega_{m, \mathrm{~d}}=0.06, K_{\mathrm{d}}=0.68$ and $C_{\mathrm{d}}=60$ for the main desorption equation. From the GAB model (13), the main sorption $\xi_{\text {a }}$ and desorption $\xi_{\mathrm{d}}$ capacity equations can be obtained by differentiating Eq. (13) relatively to $\phi$ :

$$
\xi_{\{\mathrm{d}, \mathrm{a}\}}(\phi) \equiv \frac{\partial \omega}{\partial \phi}=\frac{\omega_{m,\{\mathrm{~d}, \mathrm{a}\}} \cdot K_{\{\mathrm{d}, \mathrm{a}\}} \cdot C_{\{\mathrm{d}, \mathrm{a}\}} \cdot\left(1+K_{\{\mathrm{d}, \mathrm{a}\}}^{2} \cdot\left(C_{\{\mathrm{d}, \mathrm{a}\}}-1\right) \cdot \phi^{2}\right)}{\left(1-K_{\{\mathrm{d}, \mathrm{a}\}} \cdot \phi\right)^{2} \cdot\left(1+K_{\{\mathrm{d}, \mathrm{a}\}} \cdot\left(C_{\{\mathrm{d}, \mathrm{a}\}}-1\right) \cdot \phi\right)^{2}},
$$

An important remark can be done on the GAB model to define the main adsorption and desorption equations. If one invert Eq. (13) to express $\phi$ as a function of $\omega$, two solutions can be obtained:

$$
\begin{aligned}
& \phi_{\{\mathrm{d}, \mathrm{a}\}}(\omega)= \\
& \left\{\frac{C_{\{\mathrm{d}, \mathrm{a}\}} \cdot\left(\omega-\omega_{m,\{\mathrm{~d}, \mathrm{a}\}}\right)-2 \omega+\sqrt{C_{\{\mathrm{d}, \mathrm{a}\}}^{2} \cdot\left(\omega-\omega_{m,\{\mathrm{~d}, \mathrm{a}\}}\right)^{2}+4 \cdot C_{\{\mathrm{d}, \mathrm{a}\}} \cdot \omega \cdot \omega_{m,\{\mathrm{~d}, \mathrm{a}\}}}}{2 \cdot \omega \cdot\left(C_{\{\mathrm{d}, \mathrm{a}\}}-1\right) \cdot K_{\{\mathrm{d}, \mathrm{a}\}}}\right. \\
& \left.\frac{C_{\{\mathrm{d}, \mathrm{a}\}} \cdot\left(\omega-\omega_{m,\{\mathrm{~d}, \mathrm{a}\}}\right)-2 \omega-\sqrt{C_{\{\mathrm{d}, \mathrm{a}\}}^{2} \cdot\left(\omega-\omega_{m,\{\mathrm{~d}, \mathrm{a}\}}\right)^{2}+4 \cdot C_{\{\mathrm{d}, \mathrm{a}\}} \cdot \omega \cdot \omega_{m,\{\mathrm{~d}, \mathrm{a}\}}}}{2 \cdot \omega \cdot\left(C_{\{\mathrm{d}, \mathrm{a}\}}-1\right) \cdot K_{\{\mathrm{d}, \mathrm{a}\}}}\right\} .
\end{aligned}
$$

From a physical point of view, the correct solution is the one ensuring $\phi \geqslant 0$, if it exists. Finally, the surface coefficient are set to $h_{0}=5 \mathrm{~W} /\left(\mathrm{m}^{2} \cdot \mathrm{K}\right)$ and the emissivity to $\epsilon=0.8$.

\subsection{Results for the slow cycling test}

The material is initially set to a relative humidity $\phi=0.49$ and temperature $T=23{ }^{\circ} \mathrm{C}$, after being dried. The slow cycling test consists in submitting the sample to a step of relative humidity at $\phi=0.75$ and then at $\phi=0.33$. The measured ambient relative humidity is shown in Figure 4(a). At the bottom, the vapor flux is assumed to vanish and a NEUMANN boundary condition (7) is defined in the mathematical model. The measured temperature varies around $23{ }^{\circ} \mathrm{C}$ in the ambient air and at the bottom surface, as illustrated in Figures 4(b) and 4(c).

The numerical model described in Section 3 is used to compute the numerical solutions of temperature, relative humidity, moisture content and sorption capacity in the material. The discretisation parameters are set to $\Delta t=3.6 \mathrm{~s}$ and $\Delta x=1 \mathrm{~mm}$. This choice ensures to compute a sufficiently accurate solution of the mathematical problem. Since the time step is lower than the time interval of measurement, a linear interpolation is performed between two measured data of the boundary conditions. The numerical parameters of the hysteresis model equals to $\beta_{\mathrm{a}}=0.36 \mathrm{~h}^{-1}$ and $\beta_{\mathrm{d}}=1.79 \mathrm{~h}^{-1}$.

The results obtained with the numerical models are compared to the experimental data. Figure 5 gives the time variation of the total moisture content in the material. A good agreement can be noticed among the experimental observations and numerical results. The results are compared to the one obtained with a simulation without hysteresis, considering only the main adsorption equation $\omega=\omega_{\mathrm{a}}(\phi)$ and $\xi=\xi_{\mathrm{a}}(\phi)$ in the mathematical model. As expected, the solutions of the numerical models with and without hysteresis are identical during the adsorption phase. Then, during the desorption phase, some discrepancies appear. 
The model without hysteresis underestimate the desorption process. According to Table 1, the relative error is around $2 \%$ and $3 \%$ for the case with and without hysteresis, respectively. The probability density functions of the error between the experimental observations and the numerical predictions is computed using the kernel smoothing function for the extrapolation [38]. It is shown in Figure 6. The mean error is $\mathcal{E}\left(\omega_{\text {tot }}\right)=4 \cdot 10^{-3}$. In addition, the predictions of the model underestimates slightly the experimental measurements.

During the time interval $t \in[0,216] \mathrm{h}$, the material is submitted to an adsorption phase. The moisture content computed by the Bang-Bang model follows the main adsorption curve $\Omega_{\mathrm{a}}$, as illustrated in Figure 7(a). It is consistent since the initial state of the material corresponds to a moisture content given by the main adsorption curve $\Omega_{\mathrm{a}}$. At $t=216 \mathrm{~h}$, the material is submitted to a desorption phase. The hysteresis effects can be noted in Figure 7 (a) where the variation of the moisture content follows a scanning desorption curve. These effects appear at $t=260 \mathrm{~h}$, or $44 \mathrm{~h}$ after the change in the boundary condition. It corresponds to the time needed for the decrease of relative humidity to reach the point $x=5 \mathrm{~cm}$ in the material. Similar observations can be made for the moisture capacity in Figure 7(b). The numerical predictions at $x=5 \mathrm{~cm}$ are compared to local measurements of relative humidity and temperature in Figures $8(\mathrm{a})$ and $8(\mathrm{~b})$, respectively. There is a satisfactory agreement for the temperature, modulo the total measurement uncertainty. As reported in Table 1, the relative error is less than 1\%. However, important discrepancies are observed for relative humidity among the predictions and the experimental observations. The relative error is of the order of $13 \%$. Several reasons are mentioned in works from the literature to explain these discrepancies. Interested readers are invited to consult a complete review on the topic in [39]. Among the possible reasons, these discrepancies can be a lack in the mathematical model described in Section 2.2 to represent the physical phenomena of heat and moisture transfer. It may also arise from uncertainties in the material properties defined in Section 4.2 or by the intrusion of sensors inside the material which measures relative humidity in a small air gap as illustrated in Figure 3(b).

The evolution of the computed fields with $x$ is shown in Figure 9 for four instants during the adsorption phase $t \in\{30,40\} \mathrm{h}$ and during the desorption phase $t \in\{250,260\} \mathrm{h}$. During the adsorption phase, the relative humidity, and, as a result, the moisture content and the sorption capacity increase according to the rise of ambient air relative humidity imposed at the boundary $x=0$. Due to coupling effects, the temperature increases slightly during this phase. Then, during the desorption phase, the fields decrease following the diminution of the ambient air relative humidity. It can be remarked that the mathematical model for the hysteresis provides smooth solutions for the moisture content and sorption capacity.

The influence of the parameter $\beta$ of the hysteresis model is investigated. Since the reliability of the model is most important during the desorption phase $t \in[216,360] \mathrm{h}$, three simulations are carried for different values of the parameter $\beta_{\mathrm{d}}$. The first one considers the adjusted parameter $\beta_{\mathrm{d}}=1.79 \mathrm{~h}^{-1}$, the second one a lower value $\beta_{\mathrm{d}}^{-}=0.1 \cdot \beta_{\mathrm{d}}=0.179 \mathrm{~h}^{-1}$ and the last a higher value $\beta_{\mathrm{d}}^{+}=10 \cdot \beta_{\mathrm{d}}=17.9 \mathrm{~h}^{-1}$. The results are shown in Figure 10(a). It enhances the local sensitivity of the total moisture content relatively to the parameter $\beta$. The hysteresis effects on the total moisture content increase with the diminution of the parameter $\beta_{\mathrm{d}}$. It can be observed in Figure 10(b), where the computed moisture content $\omega$ is farther from the main adsorption curve $\Omega_{\mathrm{a}}$ with a lower value of parameter $\beta_{\mathrm{d}}$. Indeed, the computed moisture content is stronger "attracted" by the main desorption curve $\Omega_{\mathrm{d}}$ for this parameter (which is not plotted in the Figure due to the axes scale). As noted in Table 1, the value of the parameter $\beta_{\mathrm{d}}$ has a main influence on the absolute error of the predicted moisture content and, as a consequence, of the relative humidity. However, for the latter, the relative error with the experimental results remains important, reinforcing the hypothesis of a lack in the mathematical model of heat and moisture transfer and not in the hysteresis one. The parameter $\beta_{\mathrm{d}}$ has a slight impact on the prediction of the temperature as well.

\subsection{Results for the fast cycling test}

The second case designed to evaluate the reliability of the numerical predictions considers fast cycling experiments. The sample is submitted to alternating adsorption at $\phi=0.75$ and desorption phases at $\phi=0.33$. As a Moisture Buffer Value (MBV) test, the adsorption and desorption phases last $8 \mathrm{~h}$ and $16 \mathrm{~h}$, respectively. The measured boundary conditions are shown in Figures 11(a), 11(b) and 11(c). It is recalled that the surface at $x=10 \mathrm{~cm}$ is impermeable to moisture flows. The experiment is carried out for $10 \mathrm{~d}$. At the initial state, the relative humidity is uniform in the material at $\phi_{0}=0.508$. According 


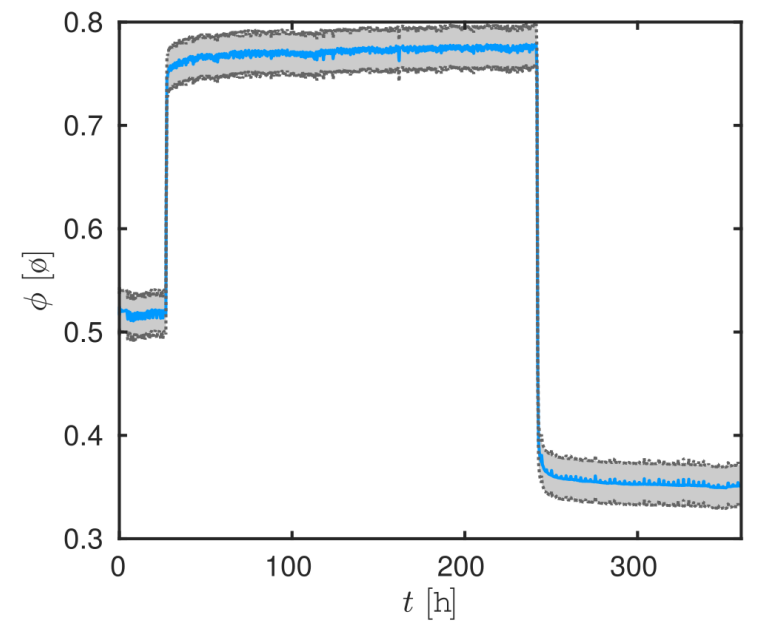

(a)

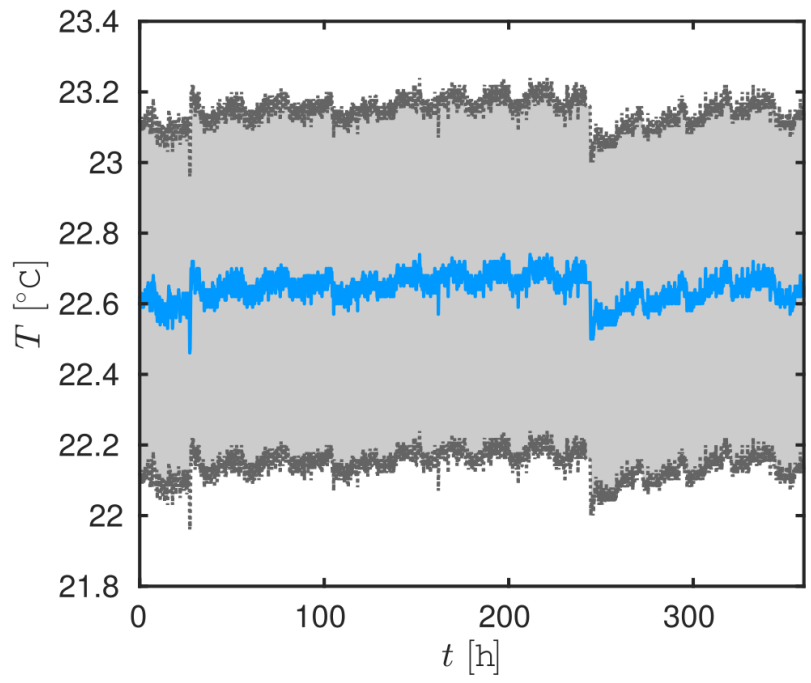

(b)

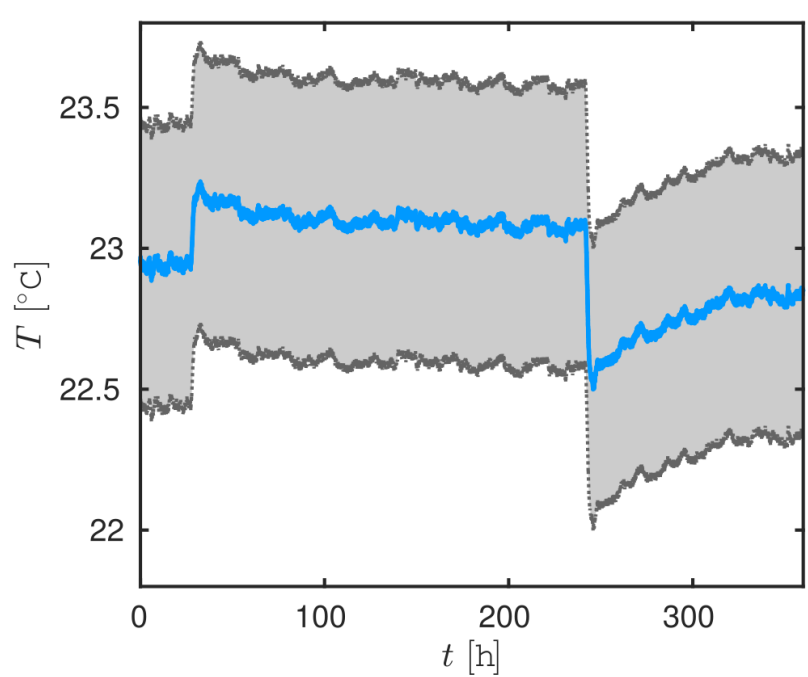

(c)

Figure 4. Measured boundary conditions for the slow cycling test of the ambient air relative humidity (a) and ambient air temperature (b) at the surface $x=0$. Measured boundary conditions of the surface temperature at $x=10 \mathrm{~cm}(\mathrm{c})$.

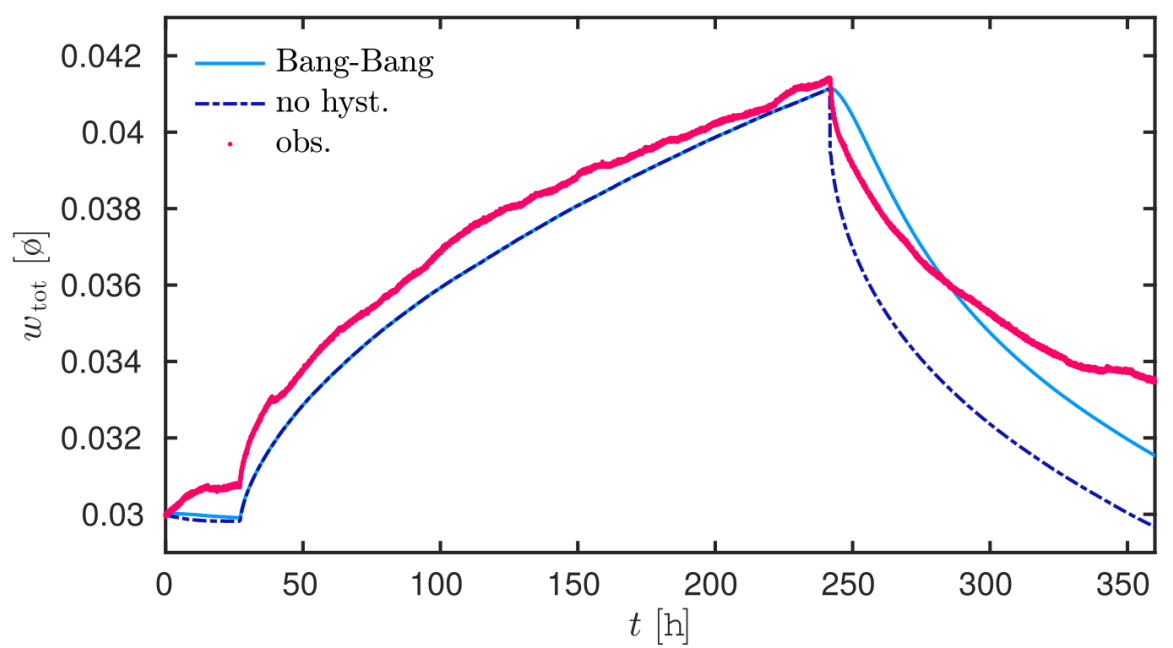

Figure 5. Comparison of the numerical predictions and experimental observations for the total moisture content $\omega_{\text {tot }}$. 


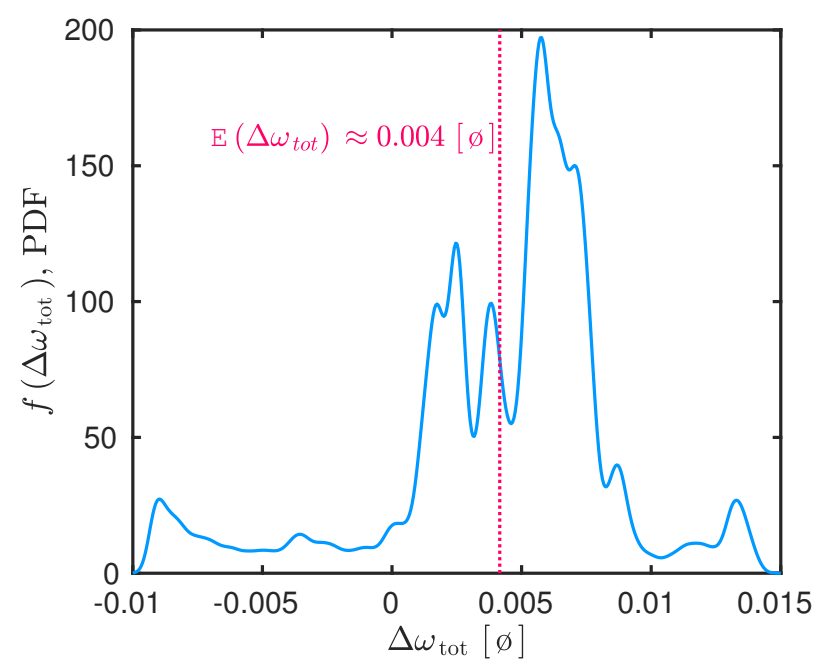

Figure 6. Probability density function of the difference between the experimental observations and the numerical predictions using the Bang-Bang model for the total moisture content $\omega_{\text {tot }}$.

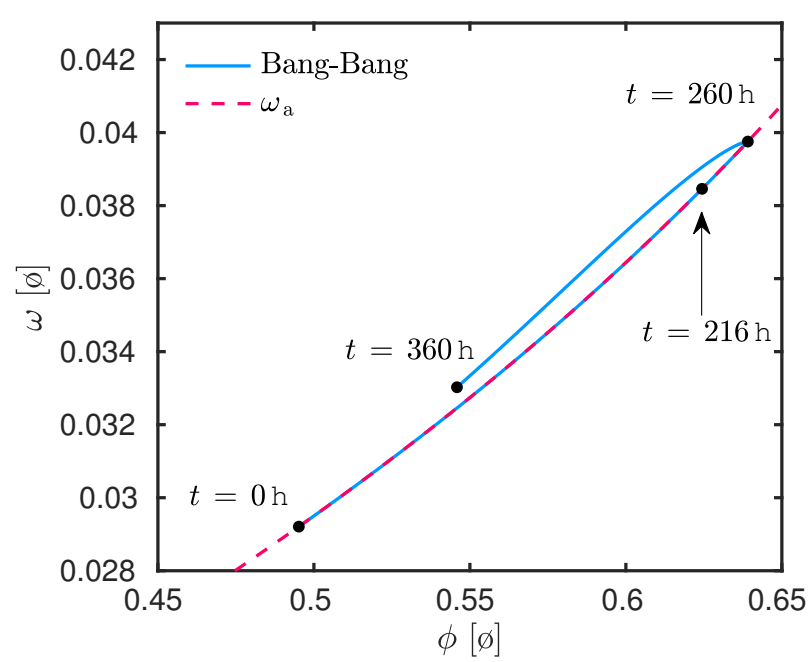

(a)

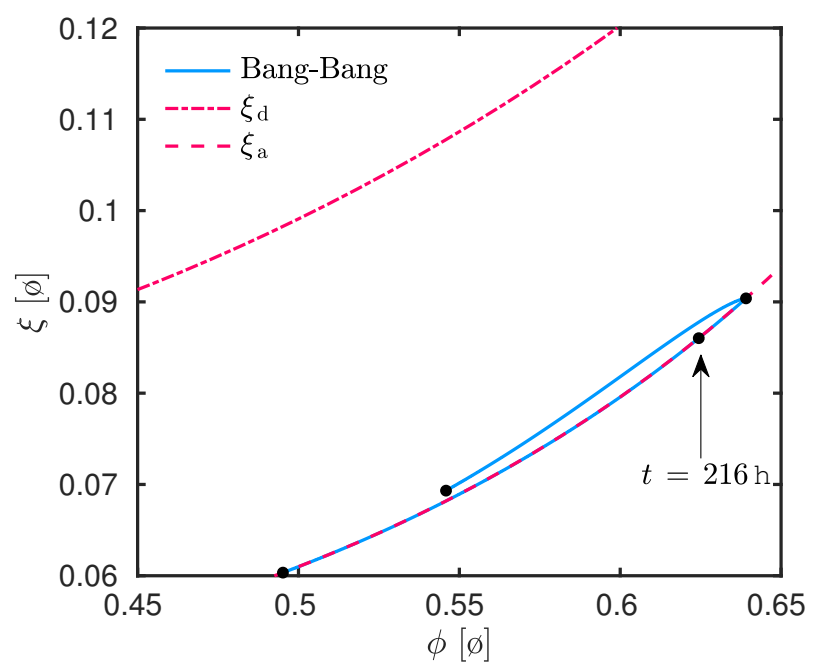

(b)

Figure 7. Evolution of the predicted local moisture content $\omega$ (a) and moisture capacity $\xi(\mathrm{b})$ at $x=5 \mathrm{~cm}$ as a function of the relative humidity $\phi$.

Table 1. Absolute error $\varepsilon_{2}$ and relative error $\varepsilon_{2, r}$ between the experimental observations and the numerical predictions for the slow cycling test.

\begin{tabular}{ccccccc}
\hline \hline & \multicolumn{2}{c}{ Water content } & \multicolumn{2}{c}{ Relative humidity } & \multicolumn{2}{c}{ Temperature } \\
Model & $\varepsilon_{2}[\varnothing]$ & $\varepsilon_{2, r}[\varnothing]$ & $\varepsilon_{2}[\mathrm{~Pa}]$ & $\varepsilon_{2, r}[\varnothing]$ & $\varepsilon_{2}\left[{ }^{\circ} \mathrm{C}\right]$ & $\varepsilon_{2, r}[\varnothing]$ \\
Bang-Bang $\beta_{\mathrm{d}}=1.79 \mathrm{~h}^{-1}$ & $6.1 \cdot 10^{-3}$ & $2.3 \cdot 10^{-2}$ & $7.7 \cdot 10^{-2}$ & $1.3 \cdot 10^{-1}$ & $1.9 \cdot 10^{-1}$ & $8.6 \cdot 10^{-3}$ \\
Bang-Bang $\tilde{\beta}_{\mathrm{d}}=0.1 \cdot \beta_{\mathrm{d}}$ & $1.7 \cdot 10^{-2}$ & $6.6 \cdot 10^{-2}$ & $7.9 \cdot 10^{-2}$ & $1.3 \cdot 10^{-1}$ & $1.9 \cdot 10^{-1}$ & $8.7 \cdot 10^{-3}$ \\
Bang-Bang $\tilde{\beta}_{\mathrm{d}}=10 \cdot \beta_{\mathrm{d}}$ & $7.7 \cdot 10^{-3}$ & $2.9 \cdot 10^{-2}$ & $7.5 \cdot 10^{-2}$ & $1.3 \cdot 10^{-1}$ & $1.9 \cdot 10^{-1}$ & $8.4 \cdot 10^{-3}$ \\
No hysteresis & & & & & & \\
$\omega=\omega_{\mathrm{a}}(\phi)$ and $\xi=\xi_{\mathrm{a}}(\phi)$ & $7.9 \cdot 10^{-3}$ & $3.1 \cdot 10^{-2}$ & $7.6 \cdot 10^{-2}$ & $1.4 \cdot 10^{-1}$ & $1.9 \cdot 10^{-1}$ & $8.7 \cdot 10^{-3}$ \\
\hline \hline
\end{tabular}




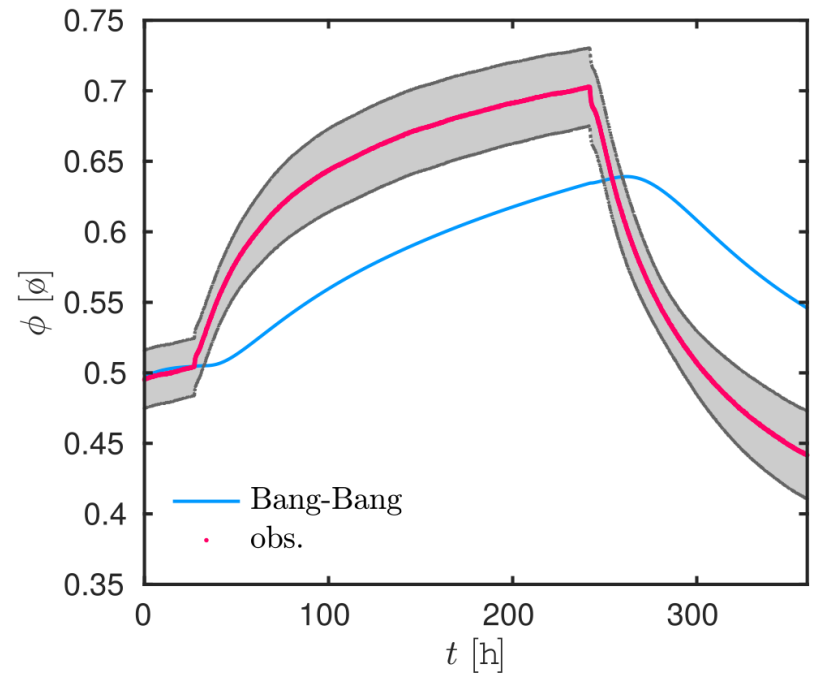

(a)

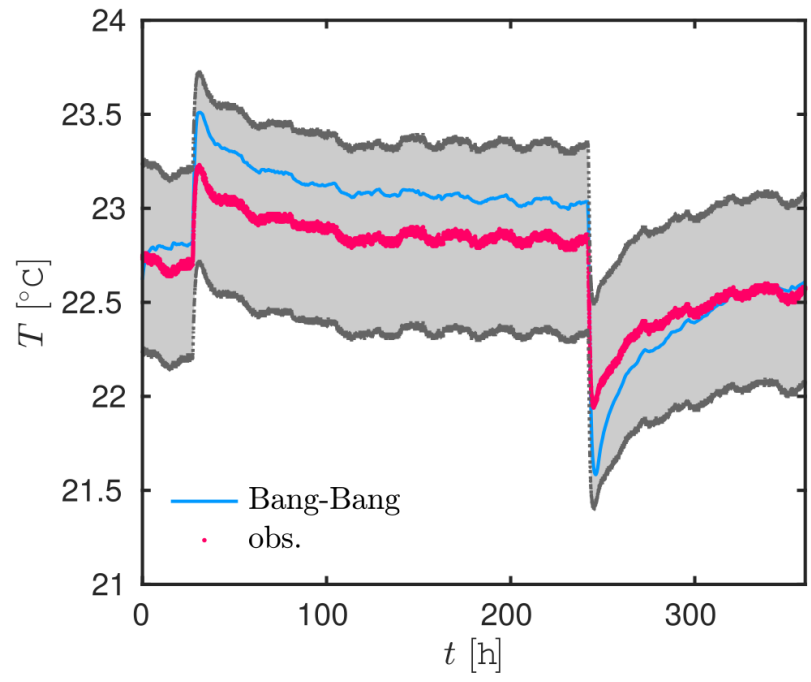

(b)

Figure 8. Evolution of the relative humidity $\phi$ (a) and the temperature $T$ (b) at $x=5 \mathrm{~cm}$ as function of time. The total uncertainties of the experimental measurements are represented with a grey shadow.

to the temperature measurements shown in Figure 11(d), the field is not uniform in the material at $t=0$. Thus, the following function has been fitted to approximate the measurement:

$$
T_{0}: x \longmapsto \tilde{T}_{0} \cdot\left(1+v_{1} \cdot \exp \left(v_{2} \cdot x\right)\right)
$$

with $\tilde{T}_{0}=296.25 \mathrm{~K}, v_{1}=-6.21 \cdot 10^{-5} \mathrm{~K}$ and $v_{2}=32.33 \mathrm{~m}^{-1}$. The accuracy of the fitting can be seen in Figure 11(d). The fields are computed using the proposed numerical model with the following disrcretisation parameters $\Delta t=3.6 \mathrm{~s}$ and $\Delta x=1 \mathrm{~mm}$. The parameters in the hysteresis model are set to $\beta_{\mathrm{a}}=0.36 \mathrm{~h}^{-1}$ and $\beta_{\mathrm{d}}=8.9 \mathrm{~h}^{-1}$.

Figure 12 shows a comparison between the computed total moisture content in the material and the measured one. A very satisfactory agreement is noted, highlighting the good reliability of the Bang-Bang model to take into account the hysteresis phenomena. As reported in Table 2, the relative error is less than $9 \%$. The hysteresis effect on the moisture content and moisture sorption capacity can be remarked in Figures 13(a) and 13(b). During the first adsorption phase $t \in[0,8] \mathrm{h}$, the moisture content follows the main adsorption curve $\Omega_{\mathrm{a}}$. Then for $t \geqslant 8 \mathrm{~h}$, the variations of the moisture content with the relative humidity follow scanning curves between the main adsorption $\Omega_{\mathrm{a}}$ and desorption $\Omega_{\mathrm{d}}$ curves. The latter cannot be seen in Figures 13(a) and 13(b) due to the axis scales. As mentioned for the previous case, the good reliability of the model depends on the value of the parameter $\beta$ in the Eqs. (4) and (5). Here, the value for the desorption phase is higher than for the previous case $\beta_{\mathrm{d}}=8.9 \leqslant 1.79 \mathrm{~h}^{-1}$. The numerical value depends certainly on the characteristic time of the physical phenomena of heat and mass transfer occurring in the material. This case deal with faster cycling test and thus with higher frequency for the variations of moisture content in the material.

The computed temperature and relative humidity at $x=1.5 \mathrm{~cm}$ are compared to experimental measurements in Figures 14(a) and 14(b). As for the previous case, important discrepancies are noted for the relative humidity. The relative error scales with $17 \%$. For temperature, there is a better agreement between the numerical predictions and the experimental observations. The computed temperature remains inside the measurement uncertainty.

Last, the profiles for the fields at different time instant are shown in Figure 15. The relative humidity increases and decreases according to the adsorption and desorption phases. Consequently, the moisture content and sorption capacity varies according to the variations of the relative humidity. It can be noted that the computed fields are smooth. The moisture penetration depth in the material can be evaluated around $3 \mathrm{~cm}$. The temperature profiles are illustrated in Figure 15(b) at the beginning of the simulation. 


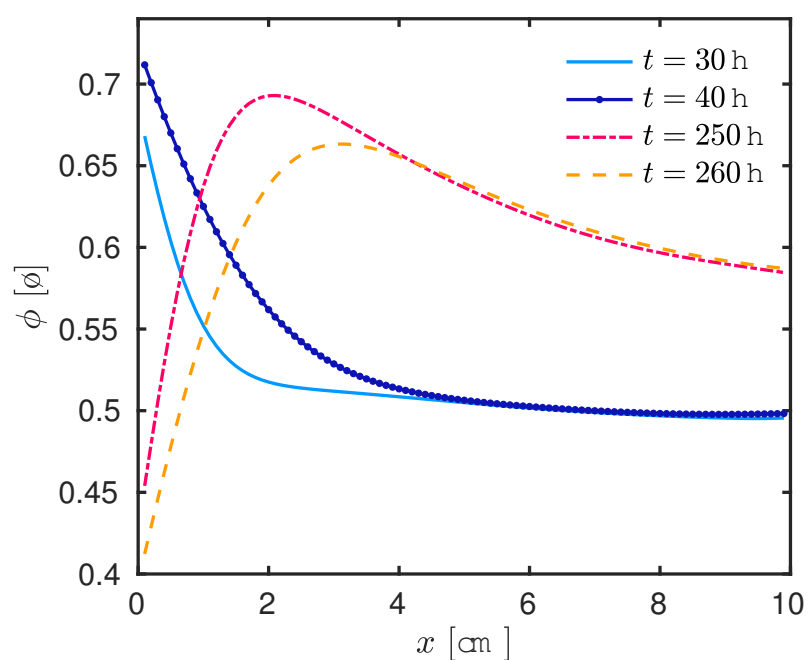

(a)

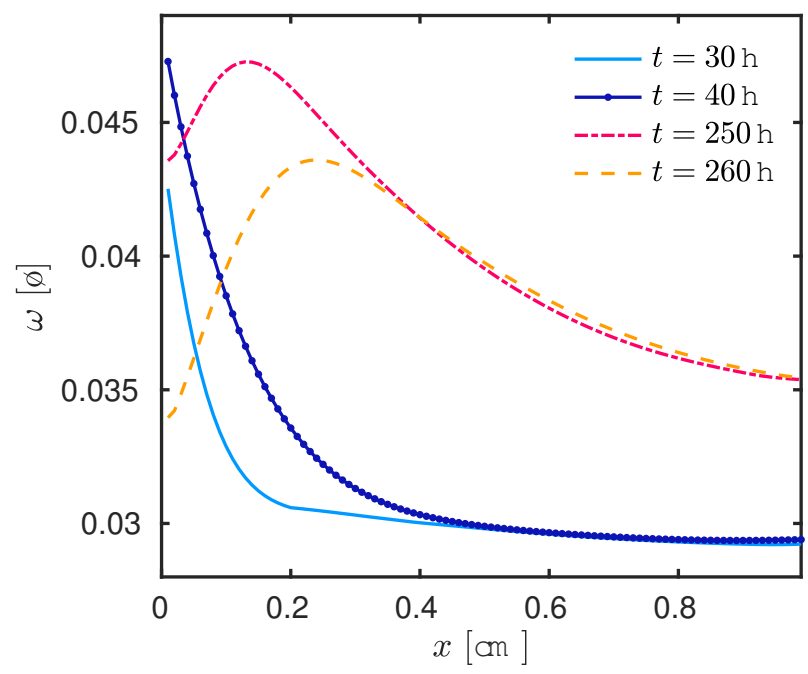

(c)

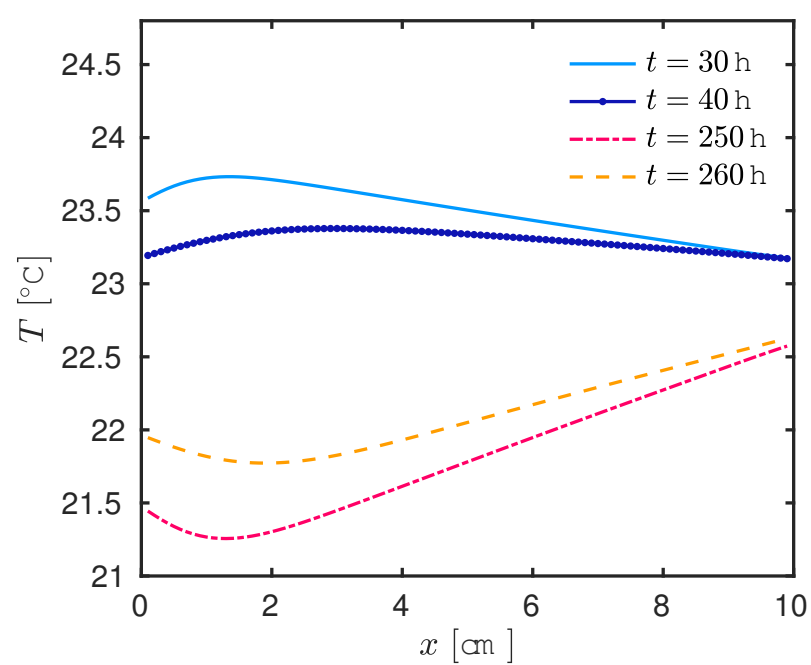

(b)

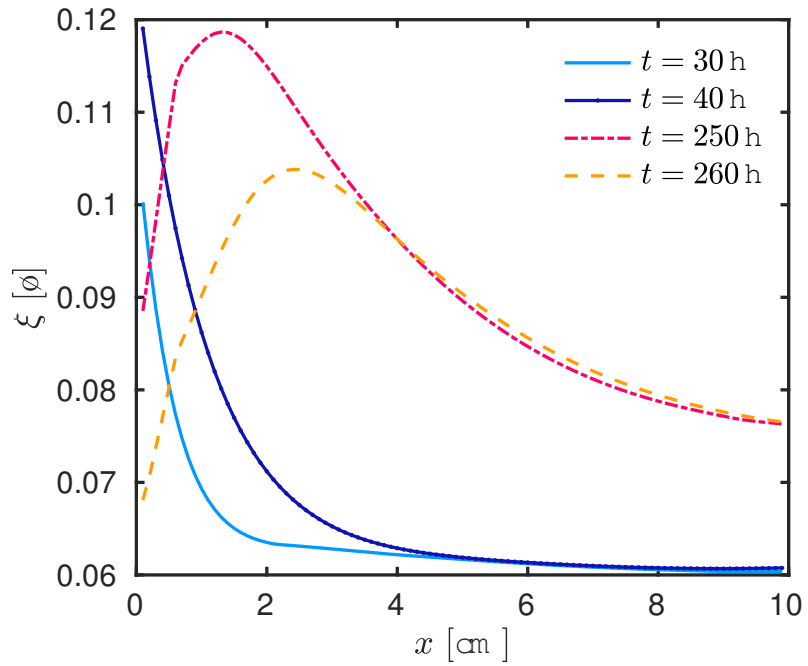

(d)

Figure 9. Variation of the relative humidity $\phi$ (a), of the temperature $T$ (b), of the moisture content $\omega$ (c) and of the moisture sorption capacity $\xi(\mathrm{d})$ as a function of the spatial coordinate $x$. 


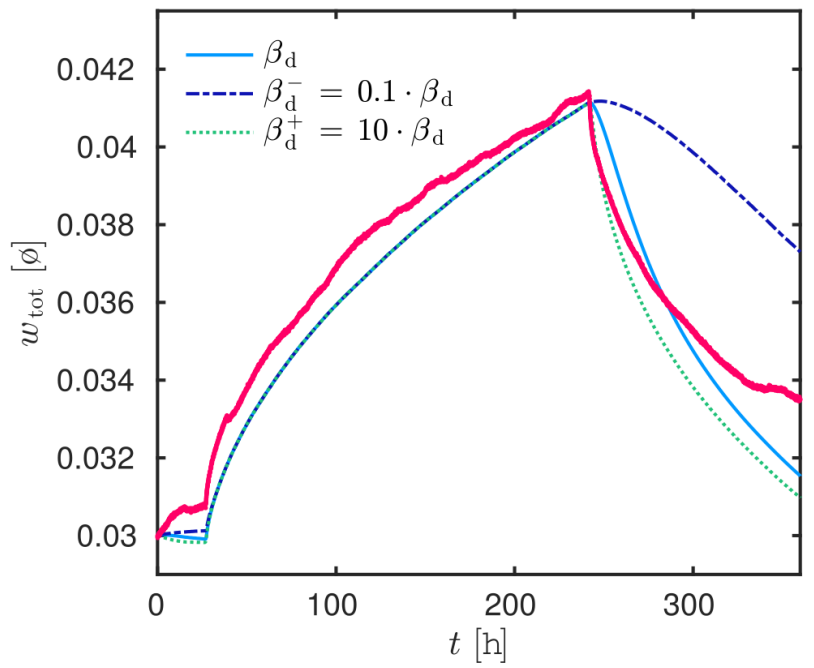

(a)

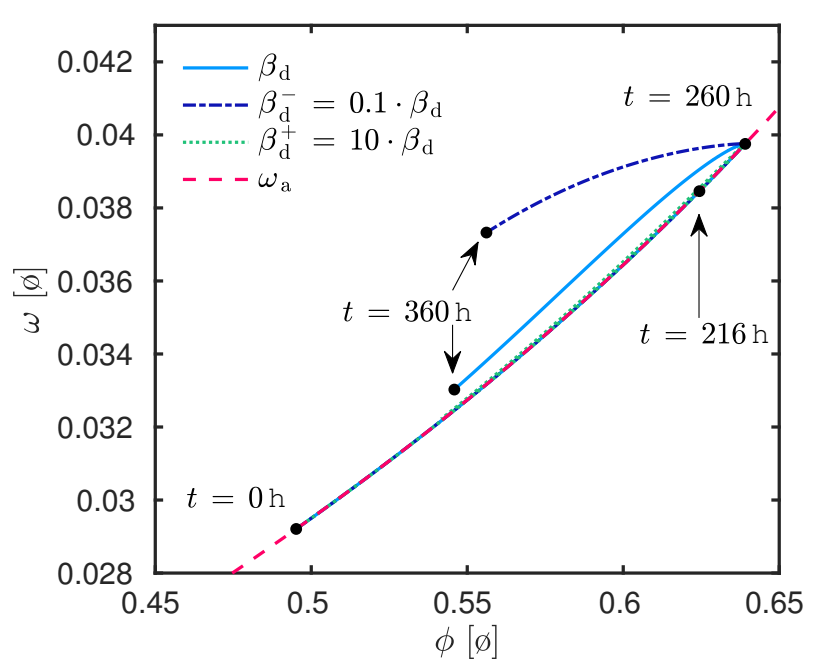

(b)

Figure 10. Influence of the parameter $\beta_{\mathrm{d}}$ on the numerical predictions of the total (a) and local (b) moisture content at $x=5 \mathrm{~cm}$.

Table 2. Absolute error $\varepsilon_{2}$ and relative error error $\varepsilon_{2, r}$ between the experimental observations and the numerical predictions for the slow cycling test.

\begin{tabular}{ccccccc}
\hline \hline Model & \multicolumn{2}{c}{ Water content } & Relative humidity & Temperature \\
& $\varepsilon_{2}[\varnothing]$ & $\varepsilon_{2, r}[\varnothing]$ & $\varepsilon_{2}[\mathrm{~Pa}]$ & $\varepsilon_{2, r}[\varnothing]$ & $\varepsilon_{2}\left[{ }^{\circ} \mathrm{C}\right]$ & $\varepsilon_{2, r}[\varnothing]$ \\
Bang-Bang $\beta_{\mathrm{d}}=8.9 \mathrm{~h}^{-1}$ & $1.9 \cdot 10^{-3}$ & $8.7 \cdot 10^{-2}$ & $8.6 \cdot 10^{-2}$ & $1.7 \cdot 10^{-1}$ & $4.9 \cdot 10^{-1}$ & $2.1 \cdot 10^{-2}$ \\
\hline \hline
\end{tabular}

The increase of temperature in the material follows the ones of the boundary condition shown in Figure 11(b). Comparing to moisture content, the heat penetrates deeper in the material.

\subsection{Complementary remarks}

The reliability of the model of heat and mass transfer including hysteresis effects is demonstrated by comparing the numerical predictions to experimental observations from the literature. A very satisfactory agreement is observed for the total moisture content. It highlights that the model can be used for the prediction of the physical phenomena.

The hysteresis model contains two parameters $\beta_{\mathrm{a}}$ and $\beta_{\mathrm{d}}$ involved in the function $\beta(\phi)$. These parameters require to be adjusted depending on the frequency of variation of the heat and mass transfer phenomena. Moreover, the hysteresis model requires to know the main adsorption $\Omega_{\mathrm{a}}$ and desorption $\Omega_{\mathrm{d}}$ curves to determine the intermediary scanning curves. These curves may be defined using any model from literature such as for instance the VAn Genuchten [40], the GAB (as in this work) or the Brunauer, EMmetT and TELLER [41] ones among others.

In terms of numerical complexity, the hysteresis model needs to solve two additional ordinary differential equations to two partial differential equations for the heat and mass transfer. For the previous case study, the ratio computational time / time horizon of simulation is $R_{\mathrm{CPU}}=23.1 \mathrm{~min} /$ day for the numerical model with hysteresis. This ratio is only reduced to $R_{\mathrm{CPU}}=19 \mathrm{~min} /$ day for a computation with only the heat and mass transfer numerical model (the Bang-Bang model is not included). It should be remarked that these CPU time measurement have been done in the Matlab ${ }^{\mathrm{TM}}$ environement, without any particular optimization of the algorithm. Even with adding two additional equations to solve, the computational cost is moderated with the use of an ad-hoc and innovative IMEX approach. Furthermore, as highlighted in the previous case studies, for instance in Figures 5 or $9(\mathrm{c})$, the use of differential equations enables to compute smooth solutions of moisture content $\omega$ and sorption capacity $\xi$. 


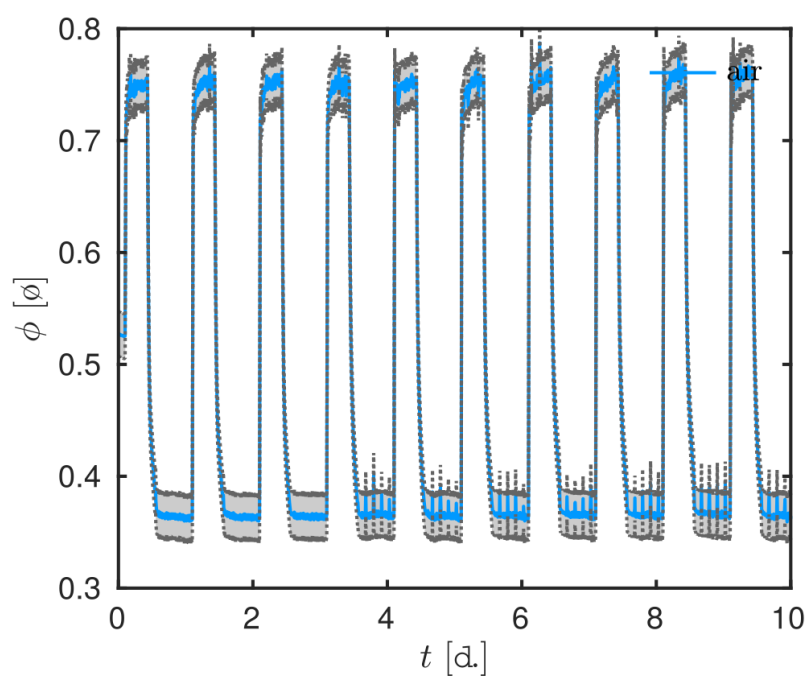

(a)

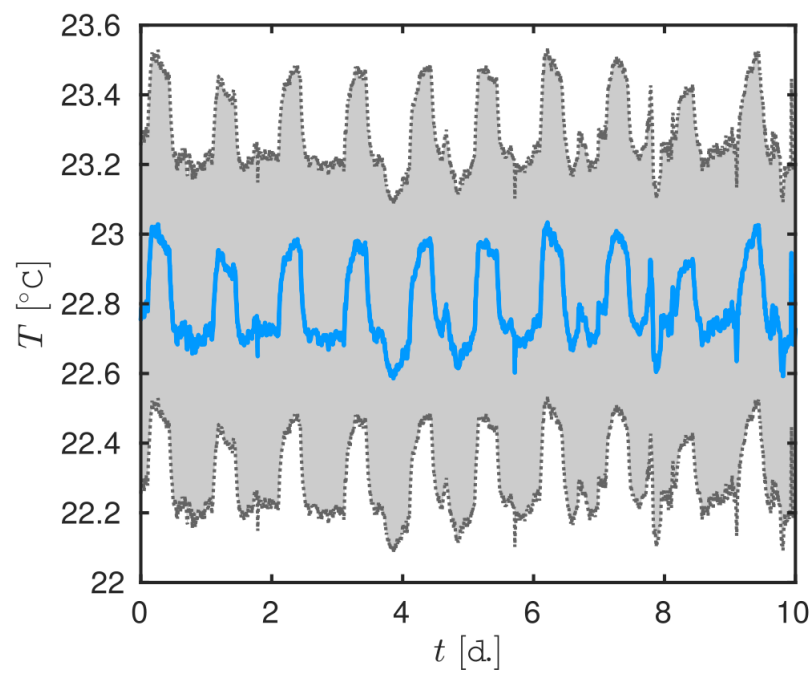

(c)

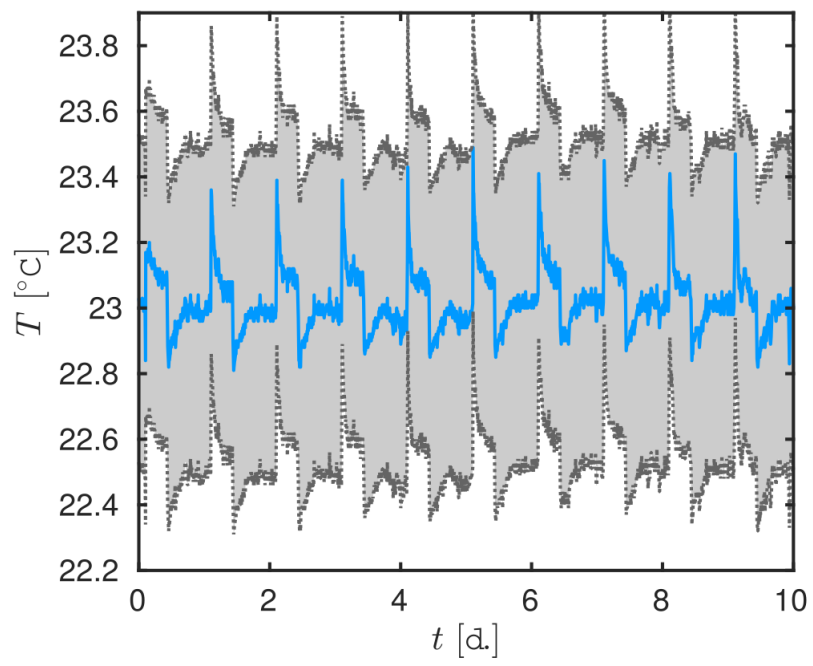

(b)

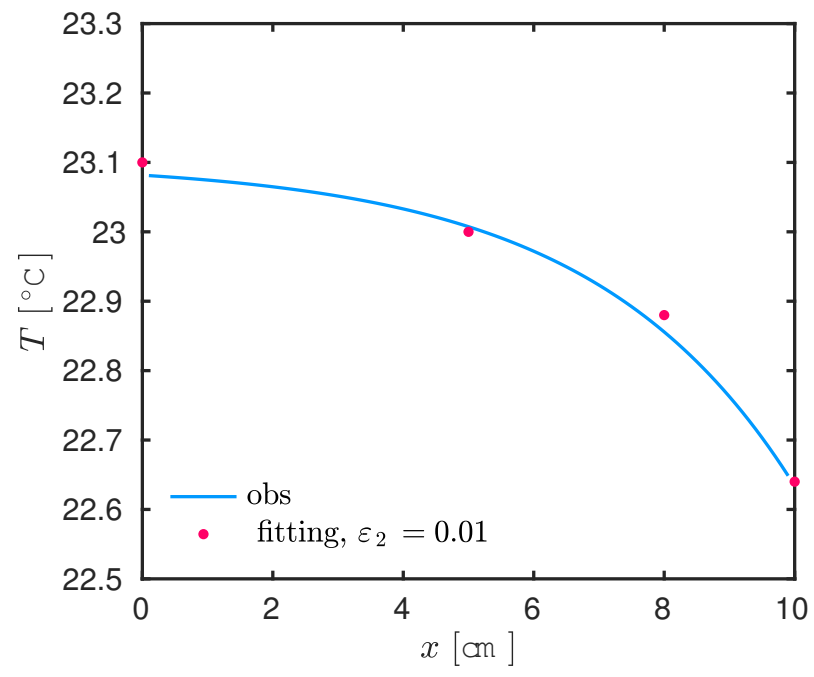

(d)

Figure 11. Measured boundary conditions for the fast cycling test of the ambient air relative humidity $\phi$ (a) and ambient air temperature $T(\mathrm{~b})$ at the surface $x=0$. Measured boundary conditions of the surface temperature at $x=10 \mathrm{~cm}$. Interpolation of the temperature initial condition (d) in the material

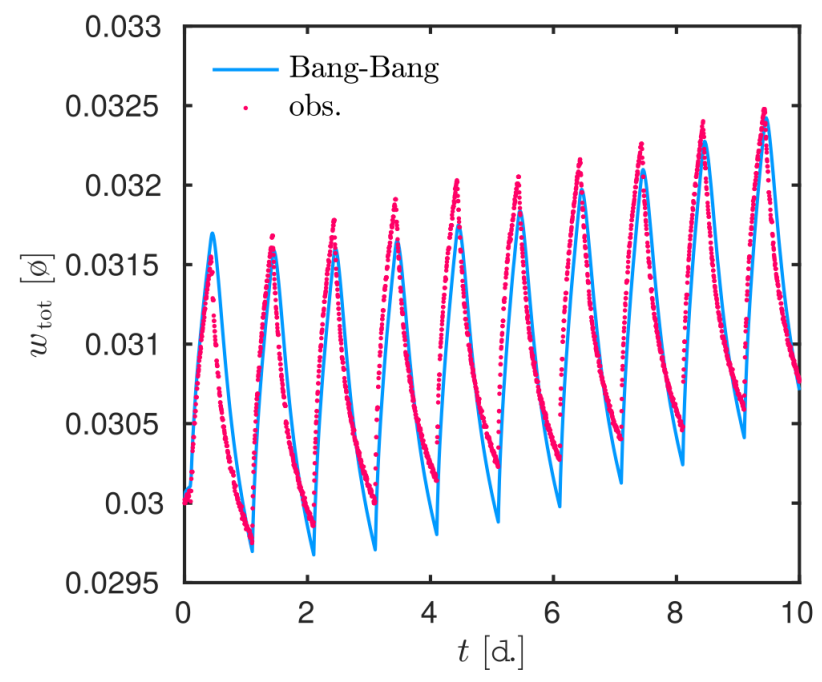

Figure 12. Comparison of the numerical predictions and experimental observations for the total moisture content $\omega_{\text {tot }}$. 


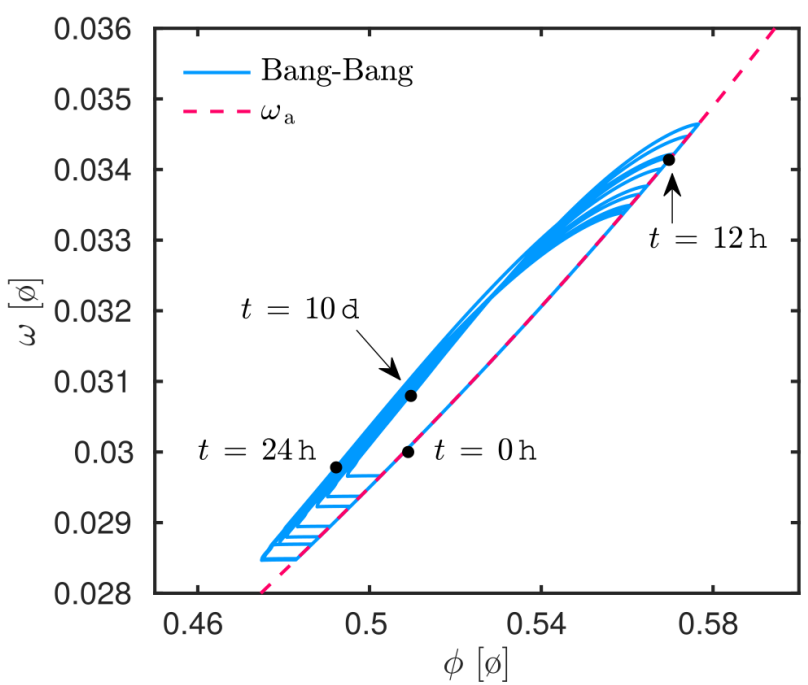

(a)

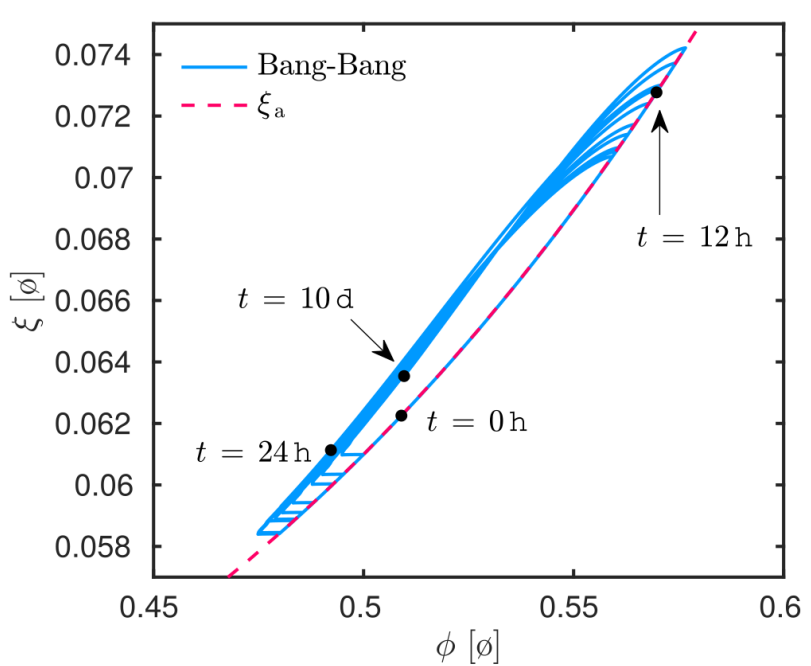

(b)

Figure 13. Evolution of the predicted local moisture content $\omega$ (a) and moisture capacity $\xi(\mathrm{b})$ at $x=1.5 \mathrm{~cm}$ in function of the relative humidity.

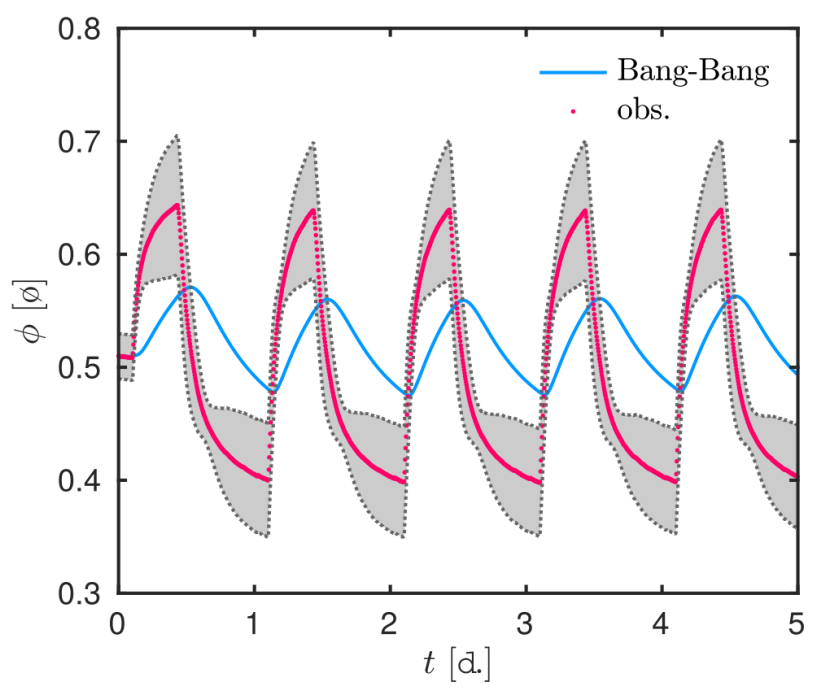

(a)

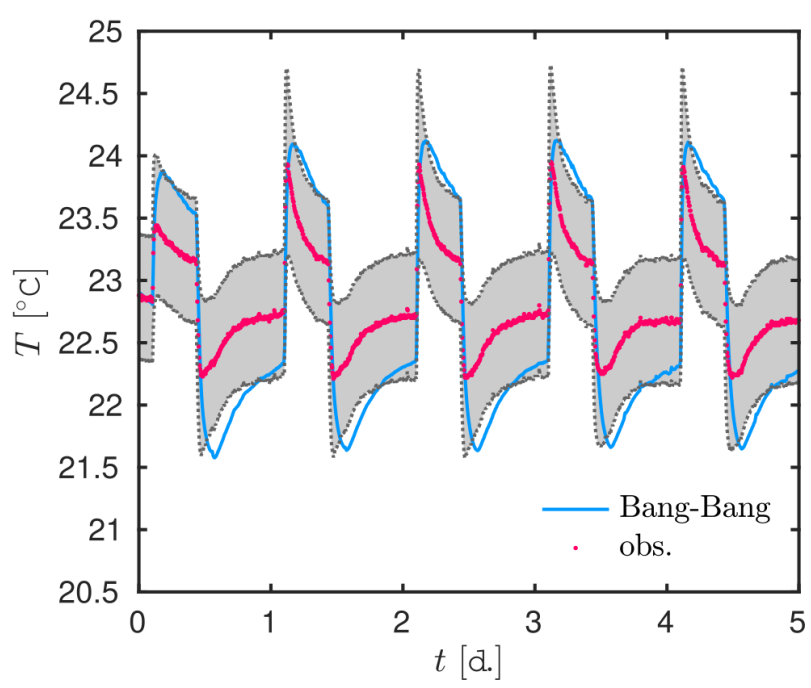

(b)

Figure 14. Evolution of the relative humidity $\phi$ (a) and the temperature (b) at $x=1.5 \mathrm{~cm}$ for the first 5 days. The total uncertainties of the experimental measurements are represented by a grey shadow. 


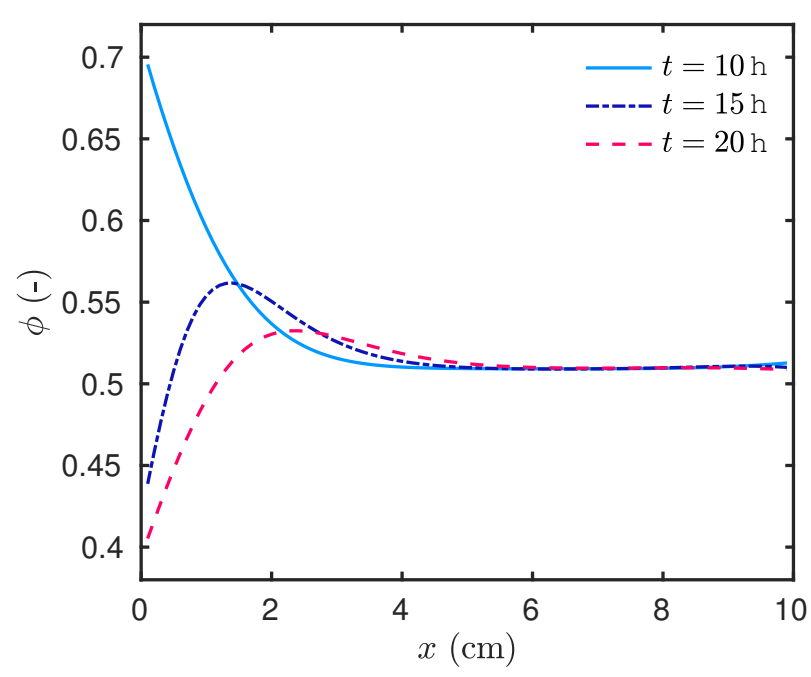

(a)

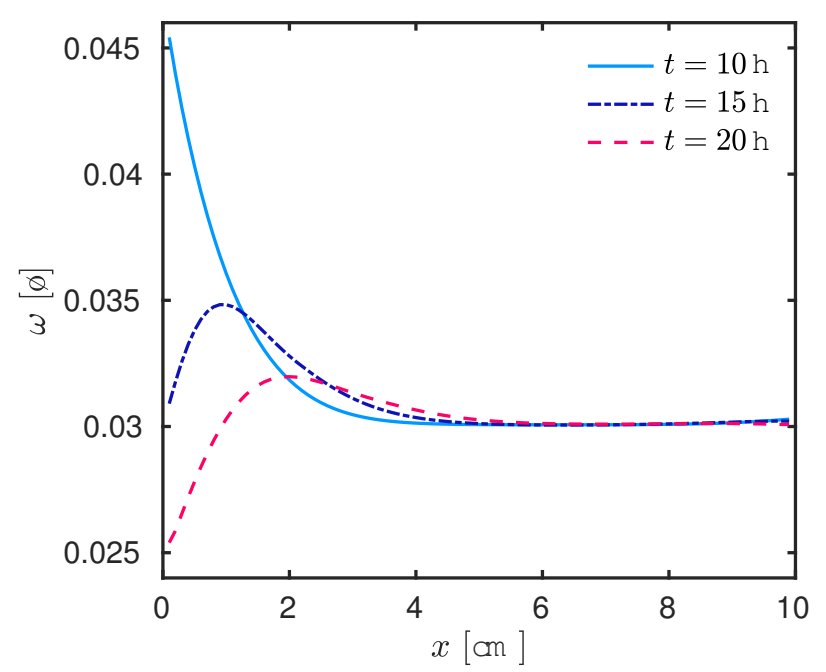

(c)

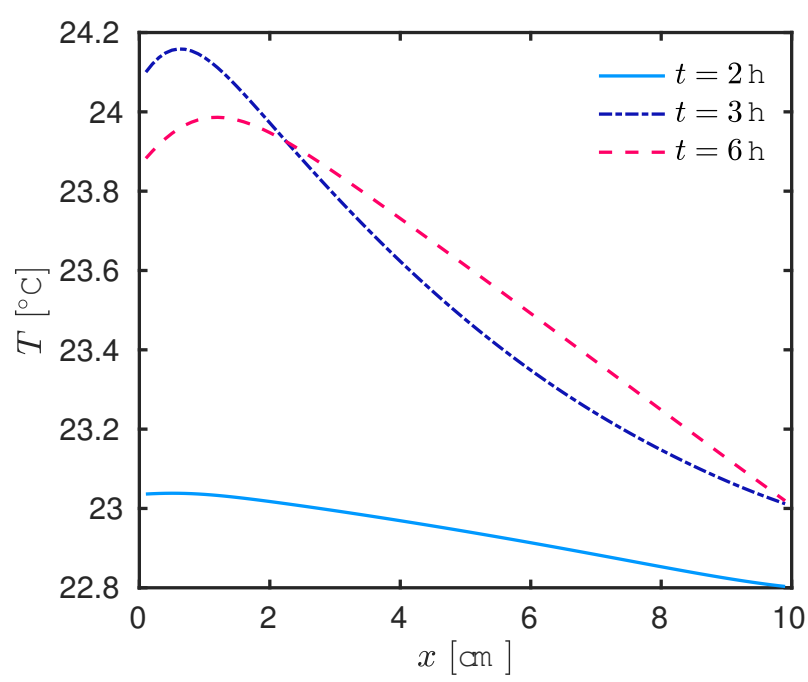

(b)

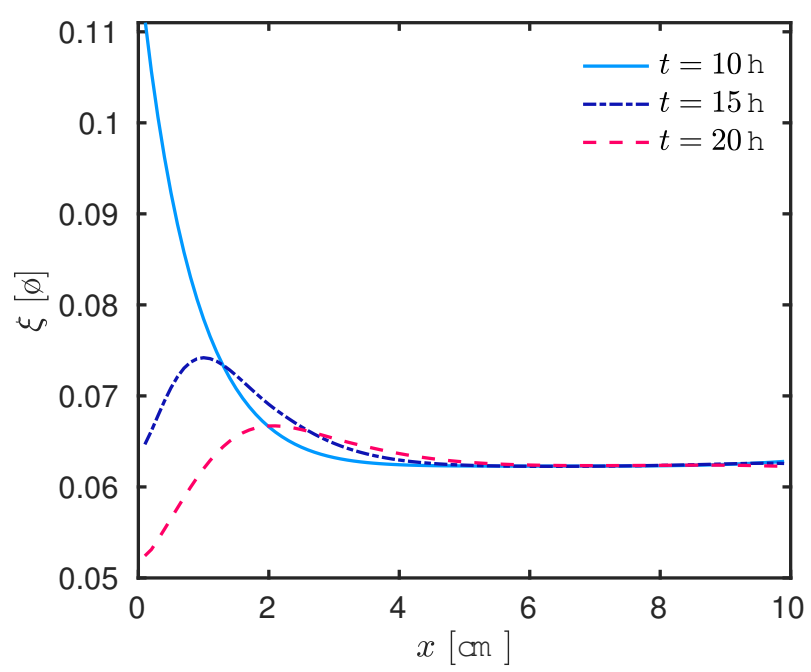

(d)

Figure 15. Variation relative humidity $\phi(\mathrm{a})$, of the temperature $T$ (b), of the moisture content $\omega(\mathrm{c})$ and of the moisture sorption capacity $\xi(\mathrm{d})$ as a function of the spatial coordinate $x$. 
These results for the Bang-Bang hysteresis model can be compared to ones from the literature. For instance, Pedersen 's model [14, 15], Mualem's one [42] and the gripped-box one [16] do not require any solution of additional partial differential equations. However, the PEDERSEN model involves three parameters to be determined depending on the material, against two for the Bang-Bang model. Moreover, its accuracy to represent the physical phenomena is questioned in [12]. One difficulty in MUALEM model is the requirement of the saturated moisture content $\omega_{s}$ to compute the intermediary scanning curves [42, Eq. (11)]. Two experimental design can be defined to obtain this value. First, it corresponds to the moisture content for the relative humidity equal to unity during the main adsorption phase $\omega_{s} \stackrel{\text { def }}{=} \omega_{\mathrm{a}}(\phi=1)$. From a practical point of view, it is a difficult task to settle an ambient conditions to $\phi=1$ with accuracy. The second design characterizes $\omega_{s}$ as the maximal possible water content in the porous. In other words, it is equal to the porosity of the material $\omega_{s} \stackrel{\text { def }}{=} \Pi$. Both experimental designs do not ensure a consistent numerical value of $\omega_{s}$. This parameter is not required either in the Bang-Bang model.

In terms of numerical complexity, at each time iteration, the gripped-box approach requires to determine the intersection between the main adsorption $\Omega_{\mathrm{a}}$ and desorption $\Omega_{\mathrm{d}}$ curves with a straight line (to compute the so-called target point [16, Section 2.1.2 and Appendix 1]). Depending on the model for the main adsorption and desorption curves, this resolution may require important computational effort. For the present case study, with the GAB model (13) for the main curves, the computation of the target points requires to solve two polynomial equations of the third degree and one of the fifth degree in $\phi$. If no exact solution can be obtained, this computation requires the use of fixed-point or NEWTON-RAPHSON algorithms for instance, which may improve significantly the computational cost of the numerical model.

\section{$5 \quad$ Influence of hysteresis on the prediction of the wall thermal loads}

The reliability of the Bang-Bang model to predict the hysteresis effects on moisture sorption has been validated in previous section by comparison with experimental data at the material scale. Now, the purpose is to perform long term simulation to investigate the influence of hysteresis effects on the wall thermal performance. Since the outside boundary conditions change rapidly, the wall is submitted to a succession of adsorption and desorption cycles. The hysteresis phenomenon may impact the heat and mass transfer process in the porous material of the wall. As a consequence, it may impact the predictions of the thermal performance and the durability of the wall.

\subsection{Case of study}

The influence of hysteresis modeling on the prediction of the hygrothermal behavior at the wall scale is studied in the case of a long-term simulation over the whole year. We consider a hemp concrete wall with a thickness $L=40 \mathrm{~cm}$ illustrated in Figure 16. The wall is submitted to climate boundary conditions on one side and to indoor boundary conditions on the other side. Figures 17(a), 17(b) and 17(c) show the boundary conditions at the two sides of the wall. The outside conditions of temperature and relative humidity, $T_{\infty}^{L}$ and $\phi_{\infty}^{L}$ correspond to the climate data used in the French thermal regulation in the Northeast region where hemp concrete is commonly used $[43,44]$. For the indoor conditions, the relative humidity has daily variations including moisture loads as illustrated in Figure $17(\mathrm{~b})$. The temperature varies between $18{ }^{\circ} \mathrm{C}$ and $23{ }^{\circ} \mathrm{C}$ during the year with a sinusoidal evolution. At the initial state, we assumed that the steady state is achieved in the wall. Thus, the initial temperature and relative humidity are given by a first order polynomial between the outside and inside conditions:

$$
\begin{aligned}
& T(x, 0)=\left(T_{\infty}^{R}(0)-T_{\infty}^{L}(0)\right) \cdot \frac{x}{L}+T_{\infty}^{L}(0), \\
& \phi(x, 0)=\left(\phi_{\infty}^{R}(0)-\phi_{\infty}^{L}(0)\right) \cdot \frac{x}{L}+\phi_{\infty}^{L}(0) .
\end{aligned}
$$

The initial conditions for the moisture content $\omega$ and sorption capacity $\xi$ are defined using a mean value between the main adsorption and desorption equations:

$$
\omega_{0}=\frac{1}{2}\left(\omega_{\mathrm{a}}\left(\phi_{0}\right)+\omega_{\mathrm{d}}\left(\phi_{0}\right)\right), \quad \xi_{0}=\frac{1}{2}\left(\xi_{\mathrm{a}}\left(\phi_{0}\right)+\xi_{\mathrm{d}}\left(\phi_{0}\right)\right) .
$$




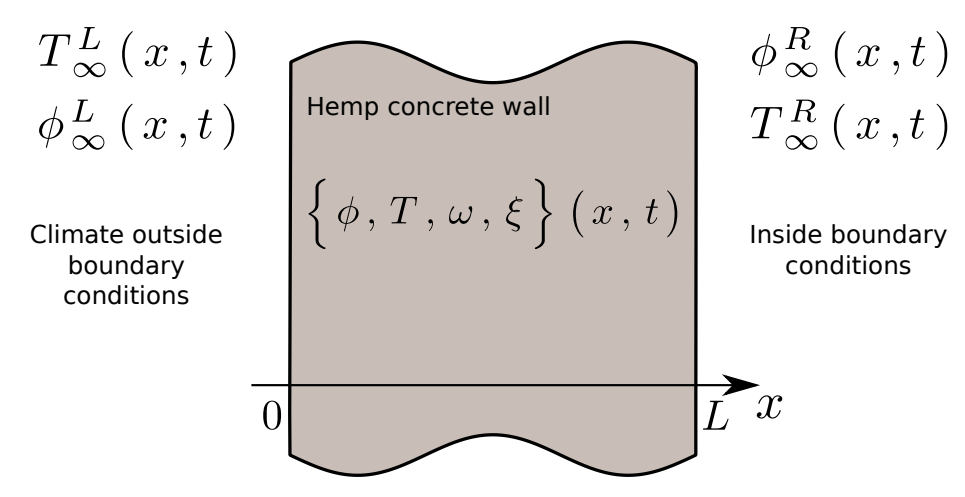

Figure 16. A schematic illustration of the case study described in Section 5.

The problem is solved using the numerical model presented in Section 3. The parameters in the hysteresis model are set to $\beta_{\mathrm{a}}=0.36 \mathrm{~h}^{-1}$ and $\beta_{\mathrm{d}}=0.54 \mathrm{~h}^{-1}$. The latter is slightly decreased compared to previous case study since the frequency of the boundary condition is higher. The discretisation parameter are set to $\Delta t=3.6 \mathrm{~s}$ and $\Delta x=1 \mathrm{~mm}$.

\subsection{Results on hysteretic behavior}

First, we focus on the evolution of the local moisture content and sorption capacity according to relative humidity at two different locations within the wall, at $x=0$ and $x=L$. The hysteresis effects can be remarked in Figures 18(a) and 18(b) for the moisture content and in Figures 18(c) and 18(d) for the sorption capacity. The computed moisture content and sorption capacity varies between the main adsorption and desorption curves. More important variations are noticed at the boundary $x=0$. Indeed, the left side of the wall is submitted to time variant moisture loads from the outside conditions. At $x=0$, the moisture content $\omega$ and sorption capacity $\xi$ oscillate between the main adsoprtion and desorption curves. Both are intermittently attracted by the main adsorption and desorption curves, depending on increase or decrease of the outside relative humidity. At the right side of the wall, $x=L$, the magnitudes of variation are lower as noted in Figure 17(b). It shows that the fields fluctuate significantly at $x=0$. On the other side, the fields remains almost constant since there the moisture loads are lower on the inside part of the wall. Similar results can be observed when analyzing the time evolution of moisture content in Figure 19(a). On the right side of the wall, the moisture content decreases fast to a value of $\omega=0.04$. It is related to the slow magnitudes of variations of relative humidity on the inside part. Indeed, Figure 22(a) gives the probability of the relative humidity $\phi$ at $x=0$ and $x=L$. The magnitudes of variation are lower at $x=L$ than $x=0$.

This computation is done for the parameter $\beta_{\mathrm{d}}=\beta_{\mathrm{d}}^{\circ}=0.54 \mathrm{~h}^{-1}$. To study the influence of this parameter on the predictions, two additional simulations are carried. The first and second computations use the parameter $\beta_{\mathrm{d}}^{-}=\frac{2}{3} \beta_{\mathrm{d}}^{\circ}=0.36 \mathrm{~h}^{-1}$ and $\beta_{\mathrm{d}}^{+}=\frac{4}{3} \beta_{\mathrm{d}}^{\circ}=0.72 \mathrm{~h}^{-1}$, respectively. The value of parameter $\beta_{\mathrm{a}}$ is not modified during these computations $\beta_{\mathrm{a}}=0.36 \mathrm{~h}^{-1}$. Figure 20(a) shows the variation of the total moisture content $\omega_{\text {tot }}$ for the three computations according to the total moisture content computed for $\beta_{\mathrm{d}}=\beta_{\mathrm{d}}^{\circ}$. The prediction of the total moisture content raises with the decreases of the parameter $\beta_{\mathrm{d}}$. A similar investigation is realized for the parameter $\beta_{\mathrm{a}}$. Three computations are carried, parameter $\beta_{\mathrm{d}}$ being fixed $\beta_{\mathrm{d}}=0.54 \mathrm{~h}^{-1}$. The first computation uses $\beta_{\mathrm{a}}=\beta_{\mathrm{a}}^{\circ}=0.36 \mathrm{~h}^{-1}$ while the two other consider higher and lower values, $\beta_{\mathrm{a}}^{-}=\frac{2}{3} \beta_{\mathrm{a}}^{\circ}=0.24 \mathrm{~h}^{-1}$ and $\beta_{\mathrm{a}}{ }^{+}=\frac{4}{3} \beta_{\mathrm{a}}^{\circ}=0.12 \mathrm{~h}^{-1}$, respectively. Figure 20(b) gives the results for the three computations. As expected, the parameter $\beta_{\mathrm{a}}$ has the opposite influence than $\beta_{\mathrm{d}}$. The prediction of the total moisture content raises with the increases of the parameter $\beta_{\mathrm{a}}$. The choice of this parameter is related to the frequency of variations of the moisture content in the material. These results confirm the possibility of using the proposed mathematical model associated with its numerical solution to predict phenomena of heat and mass transfer in building porous material 


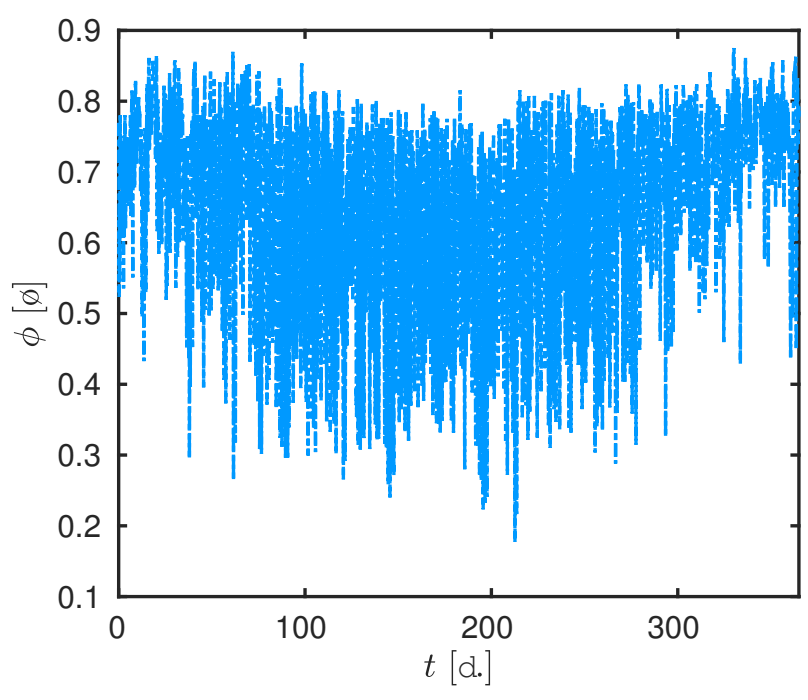

(a)

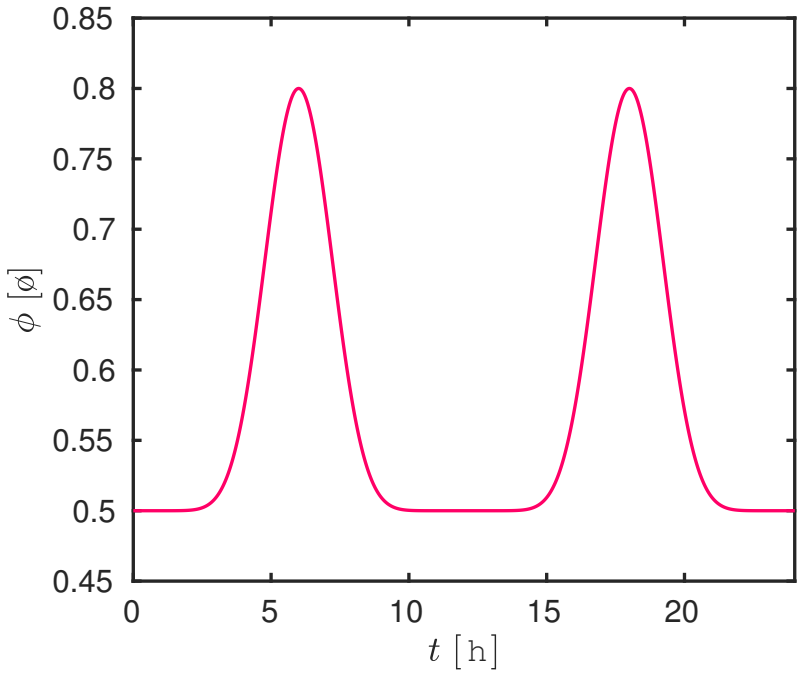

(b)

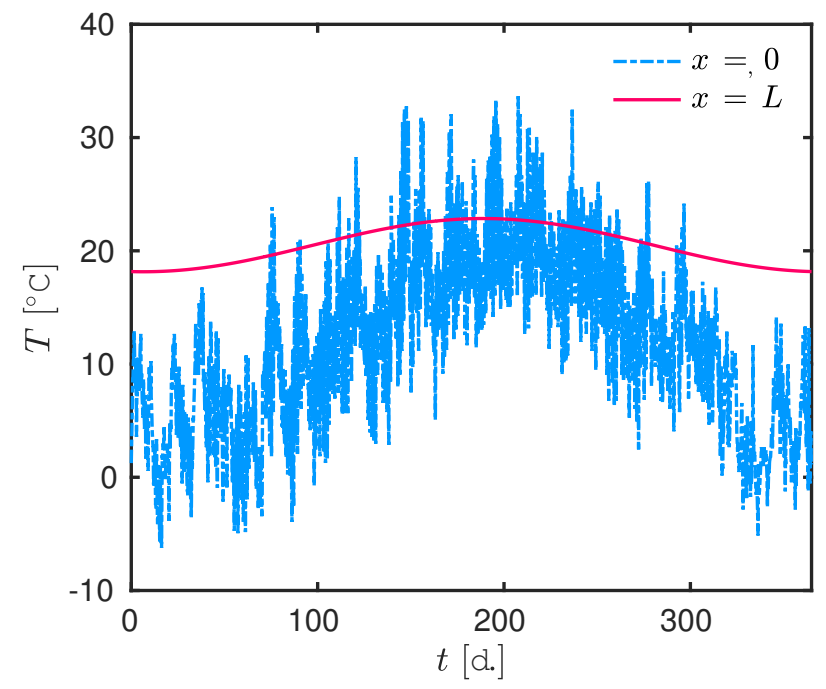

(c)

Figure 17. Evolution of the boundary conditions for outside relative humidity $\phi_{\infty}^{L}$ (a), inside relative humidity $\phi_{\infty}^{R}$ (b) and temperature (c) in long-term simulation. 


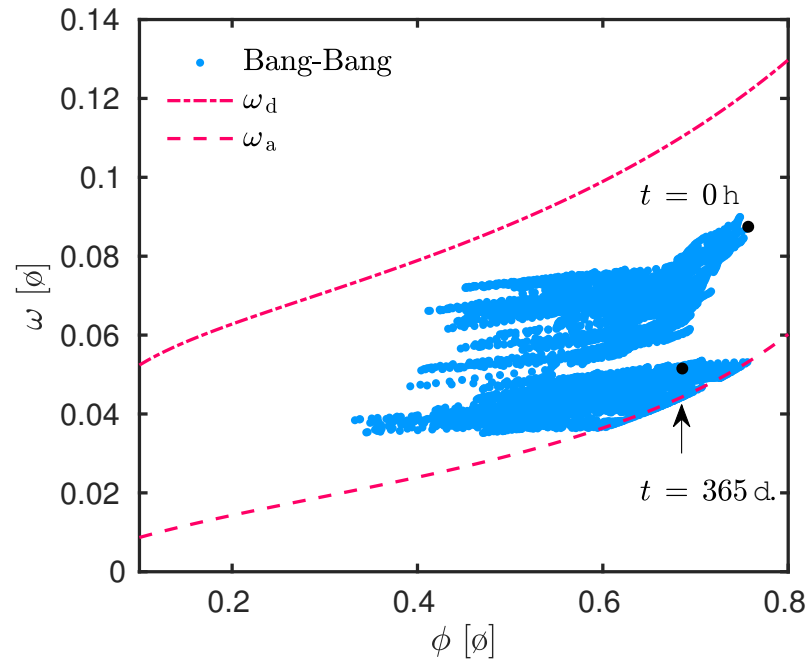

(a)

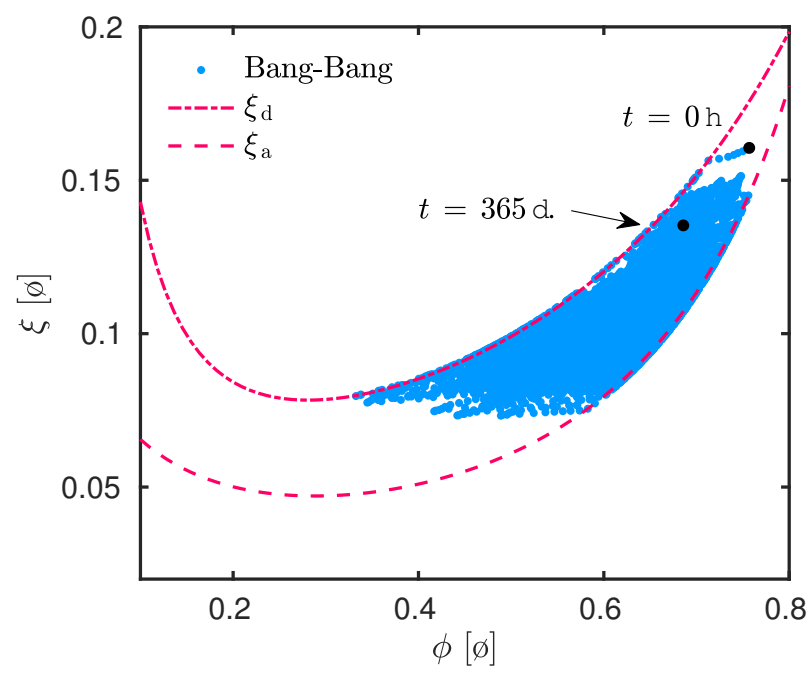

(c)

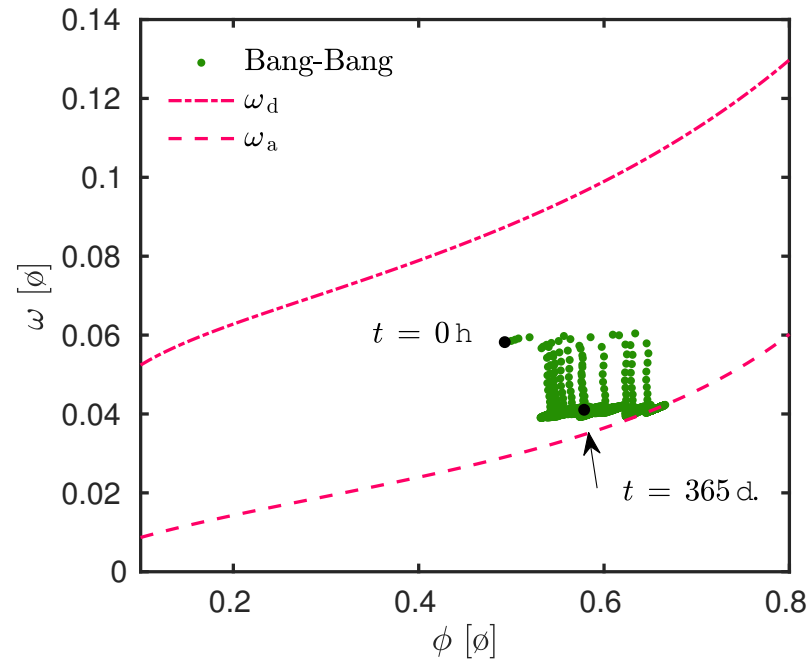

(b)

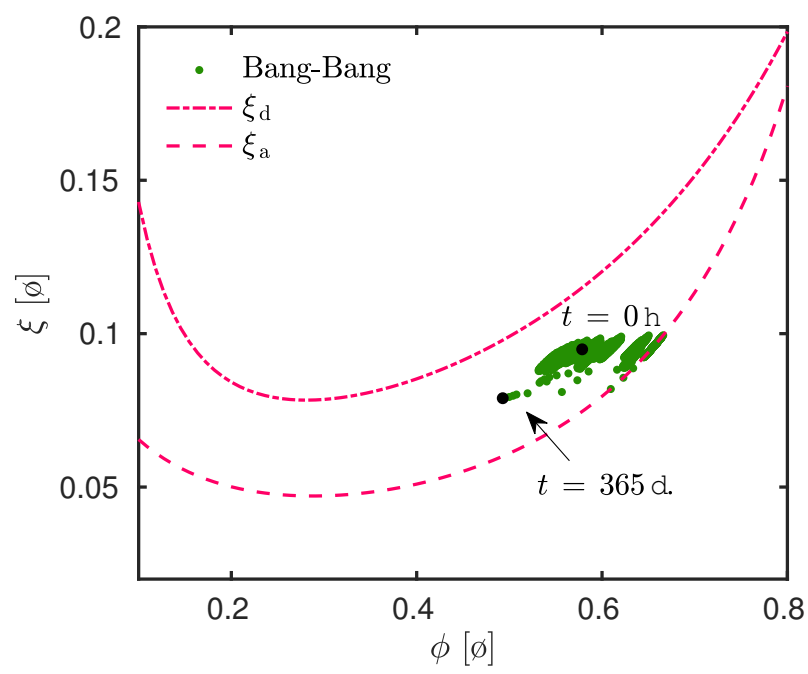

(d)

Figure 18. Evolution of the predicted local moisture content $\omega(\mathrm{a}, \mathrm{b})$ and sorption capacity $(\mathrm{c}, \mathrm{d})$ at $x=0$ $(\mathrm{a}, \mathrm{c})$ and at $x=L(\mathrm{~b}, \mathrm{~d})$ according to the relative humidity $\phi$.

with hysteresis effects on moisture content. For this, it is important to adjust the two parameters $\beta_{\mathrm{d}}$ and $\beta_{\mathrm{a}}$ according to the frequency of variation of heat and moisture inside the material. For the moment, no equation has been obtained to define the value of the parameters according to the frequency of variation.

\subsection{Complementary analysis}

To evaluate the importance of including hysteresis effects in the mathematical model to obtain reliable predictions, four different cases are simulated. (i) The first one does not include the hysteresis model. Thus Eqations (4) and (5) are omitted in the mathematical model. The moisture content and sorption capacity are directly computed using the main adsorption curve:

$$
\omega: \phi \longmapsto \omega_{\mathrm{a}}(\phi), \quad \xi: \phi \longmapsto \xi_{\mathrm{a}}(\phi) .
$$

This approach is the one used in most models from literature [1]. By comparison with other approaches, it enables to quantify the error done by omitting hysteresis effects in the model. For the sake of clarity, in next sections this approach is nominated as Model 1. (ii) The second approach, identified as Model 2, takes into account the main desorption curve:

$$
\omega: \phi \longmapsto \omega_{\mathrm{d}}(\phi), \quad \xi: \phi \longmapsto \xi_{\mathrm{d}}(\phi) .
$$




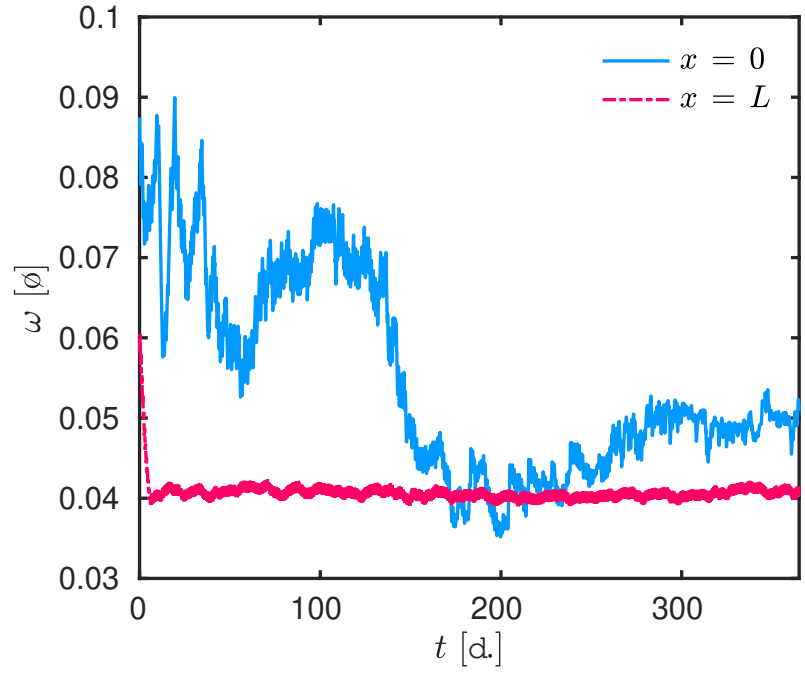

(a)

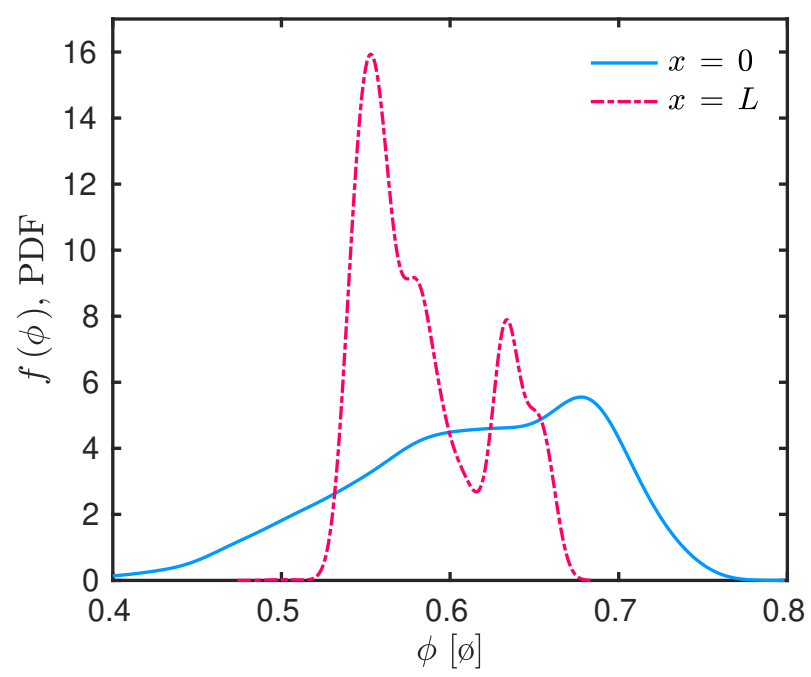

(b)

Figure 19. Time evolution of the local moisture content $\omega$ (a) and probability of the local relative humidity $\phi$ (b) at $x=0$ and at $x=L$.

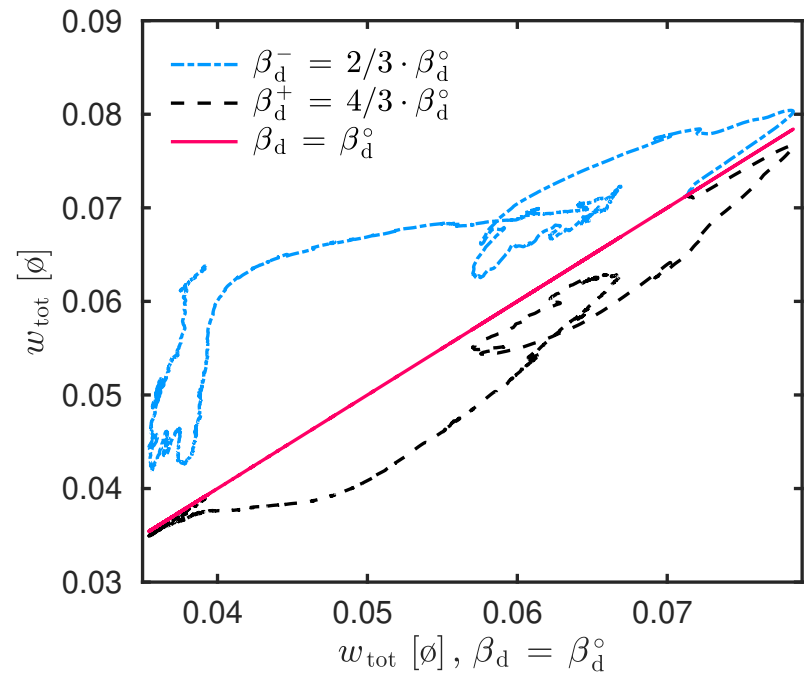

(a)

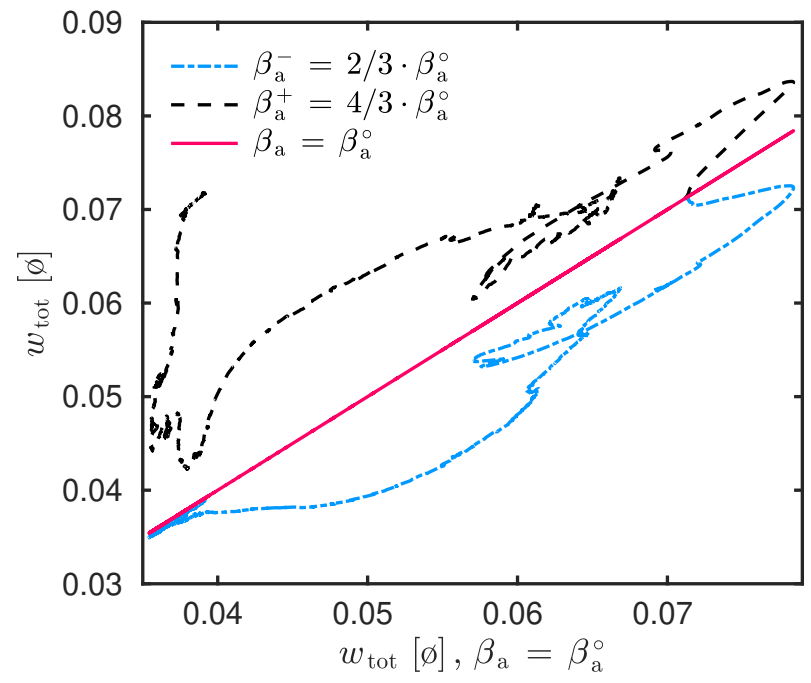

(b)

Figure 20. Evolution of the total moisture content $\omega_{\text {tot }}$ according to the parameters $\beta_{\mathrm{d}}$ (a) and $\beta_{\mathrm{a}}$ (b). 
Table 3. Synthesis of the four approaches investigated in Section 5.3

\begin{tabular}{cc}
\hline \hline Model & Hysteresis effects? \\
Model 1 & no model, given by main adsorption curve $\Omega_{\mathrm{a}}$ \\
Model 2 & no model, given by main desorption curve $\Omega_{\mathrm{d}}$ \\
Model 3 & no model, given by mean curve between main adsorption and desorption curves \\
Bang-Bang & yes, provided by Eqs. (4) and (5) \\
\hline \hline
\end{tabular}

(iii) The third approach still not include hysteresis effects and uses a mean curve between main sorption and desorption ones:

$$
\omega: \phi \longmapsto \frac{1}{2}\left(\omega_{\mathrm{a}}(\phi)+\omega_{\mathrm{d}}(\phi)\right), \quad \xi: \phi \longmapsto \frac{1}{2}\left(\xi_{\mathrm{a}}(\phi)+\xi_{\mathrm{d}}(\phi)\right) .
$$

This approach explore if the predictions of the model are sufficiently precise to avoid any extra-computational costs by including the hysteresis model. It is designated as Model 3. (iv) For the last approach, the hysteresis effects are included in the model of heat and mass transfer using the Bang-Bang model. The parameters in the hysteresis model are set to $\beta_{\mathrm{a}}=0.36 \mathrm{~h}^{-1}$ and $\beta_{\mathrm{d}}=0.54 \mathrm{~h}^{-1}$. This approach is nominated by Bang-Bang. The analysis of the results focus at $x=L$ since this boundary is in contact with the inside part of the building. A synthesis of the four approaches is given in Table 3 .

Figure 21 compares the prediction of the total moisture content $\omega_{\text {tot }}$ for the four simulation cases. Important differences are noted between the three cases without hysteresis model (Model 1, Model 2 and Model 3). As expected, the predictions for the case Model 3 are between the predictions for the ones Model 1and Model 2. Important differences in the prediction are noted when using the Bang-Bang hysteresis model, particularly for the first 100 days. Around $t=200$ days, the total moisture content corresponds to the one predicted by the approach without hysteresis using only main sorption curve $\Omega_{\mathrm{a}}$ (case Model 1). This behavior is also due to the parameter $\beta_{\mathrm{a}}$ which quantifies the attraction of the moisture content to the main sorption curve.

In Figure 22(a), the probability density function of the predicted relative humidity at $x=L$ is shown. If the mean of the prediction is rather similar, some local discrepancies are noted in the predictions of the four cases. The probability of the temperature is given in Figure 22(b) for the four different cases. The discrepancies are lower and the four cases provided similar predictions. These results are consistent. The moisture content and sorption capacity impacts directly the computation of the relative humidity $\phi$ in Eq. (1b) through coefficients $c_{\mathrm{M}}$ and $k_{\mathrm{M}}$. For the heat transfer equation (1a), the impact of moisture content and sorption capacity is lower. Indeed, the coupling parameter $\gamma$, which translates the impact of the mass transfer on the heat equation, scales with $\gamma=2.2 \cdot 10^{-3}$. At the reference condition $\left(T^{\circ}=280 \mathrm{~K}\right.$ and $\phi^{\circ}=1$ ), the latent heat flux is 3 order lower than the sensible one. The impact of mass transfer on heat one is rather low.

The time variation of the fluxes are shown in Figures $23(\mathrm{a})$ and $23(\mathrm{~b})$ for the $48^{\text {th }}$ day. In addition, the probability density function of the heat and mass fluxes computed over the whole time interval are given in Figures 23(c) and 23(d). Important discrepancies are revealed for both predictions. The cases Model 1 and Model 2 overestimates and underestimates the heat and mass fluxes, respectively. The approach Model 3, which uses the mean between the main adsorption and desorption curves, has closer predictions of the heat flux $j_{\mathrm{q}}$ with the model including the hysteresis effects. Nevertheless, if one requires a reliable model for the prediction of mass flux, it is of capital importance to include the hysteresis effects.

In terms of computational cost of the four different cases. For the approaches Model 1, Model 2 and Model 3, the computational ratio scales with $R_{\mathrm{CPU}}=11.2 \mathrm{~min} /$ day. For the Bang-Bang model, which includes hysteresis effects, the ratio increases slightly to $R_{\mathrm{CPU}}=12.8 \mathrm{~min} /$ day. Thanks to an efficient numerical model the computational efforts are saved. A more reliable model for the predictions of the heat and mass transfer in building porous materials is obtained at the price of small increase of the computation time. 


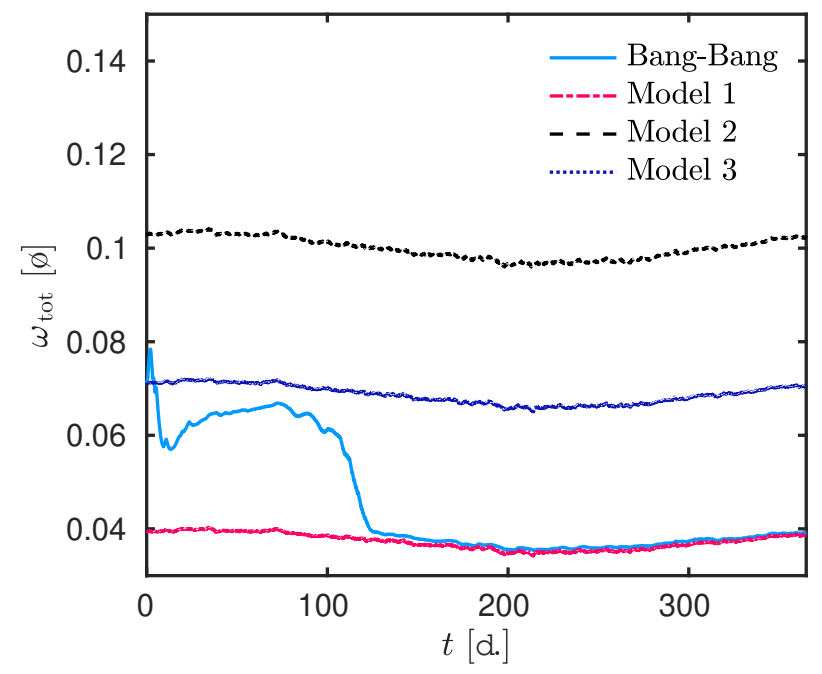

Figure 21. Time evolution of the total moisture content $\omega_{\text {tot }}$ in the wall for the four different simulation cases.

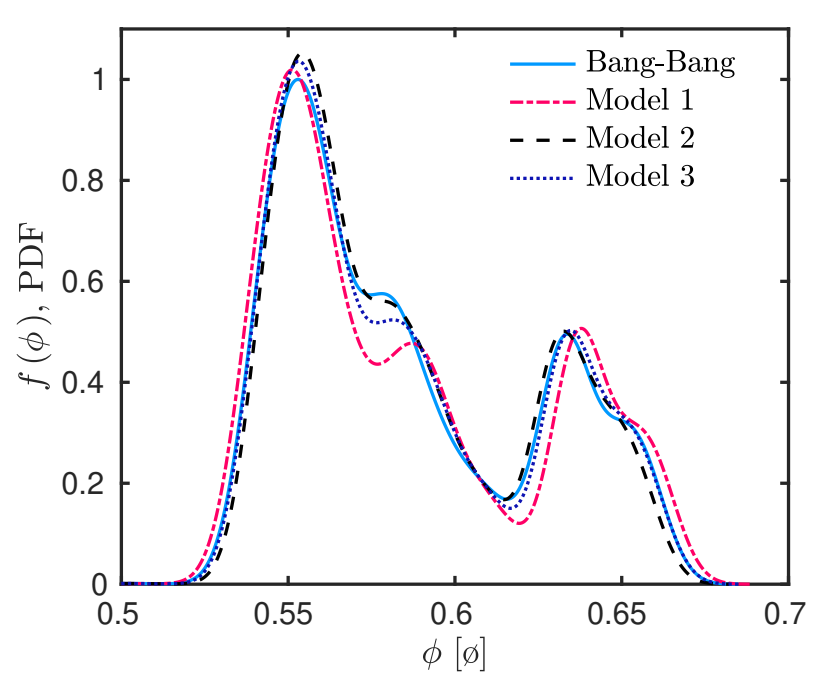

(a)

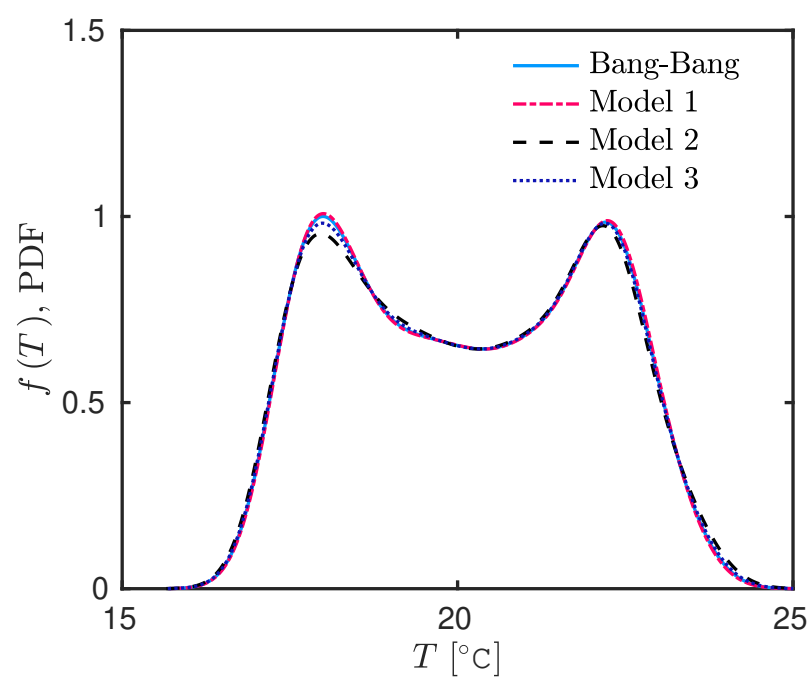

(b)

Figure 22. Probability density function of the relative humidity $\phi$ (a) and of the temperature $T$ (b) at $x=L$ for the four different simulation cases. 


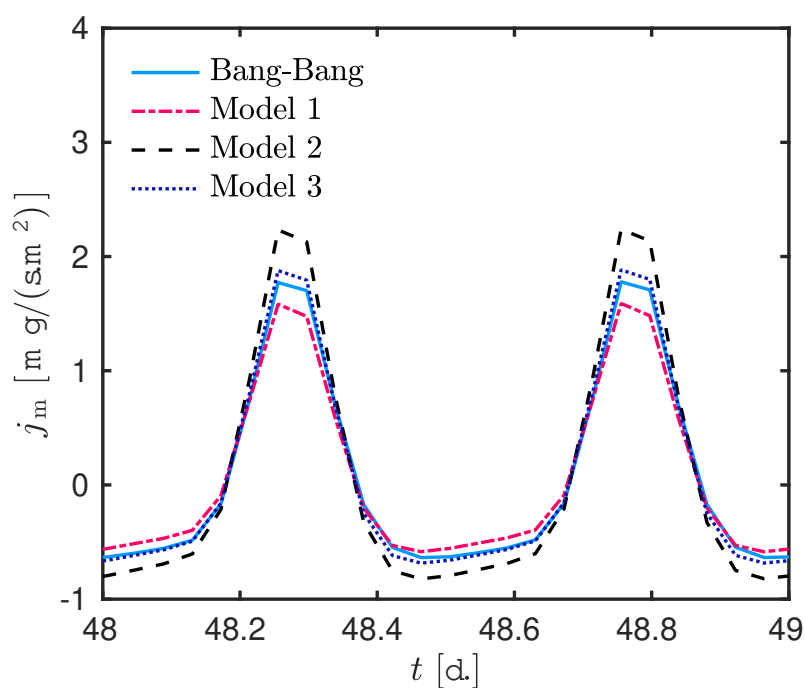

(a)

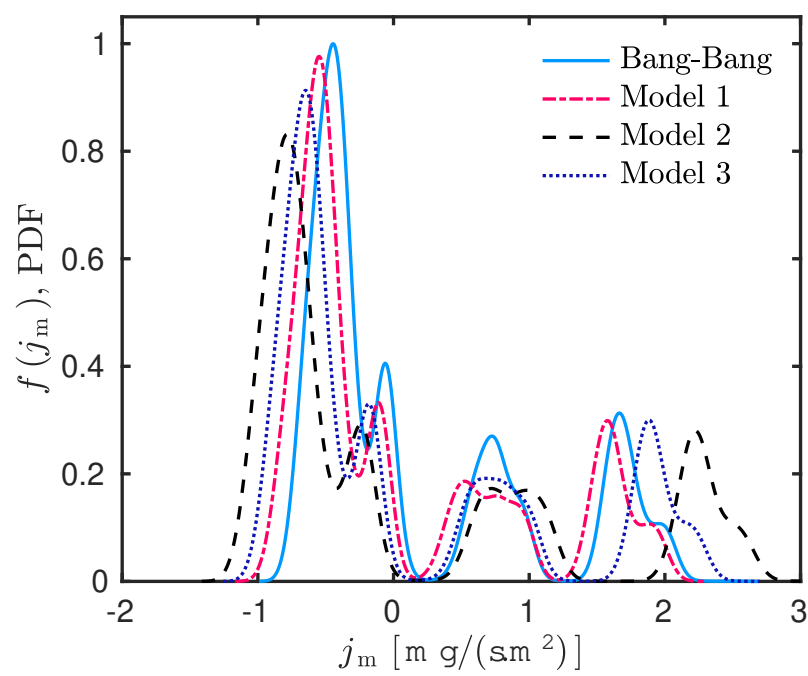

(c)

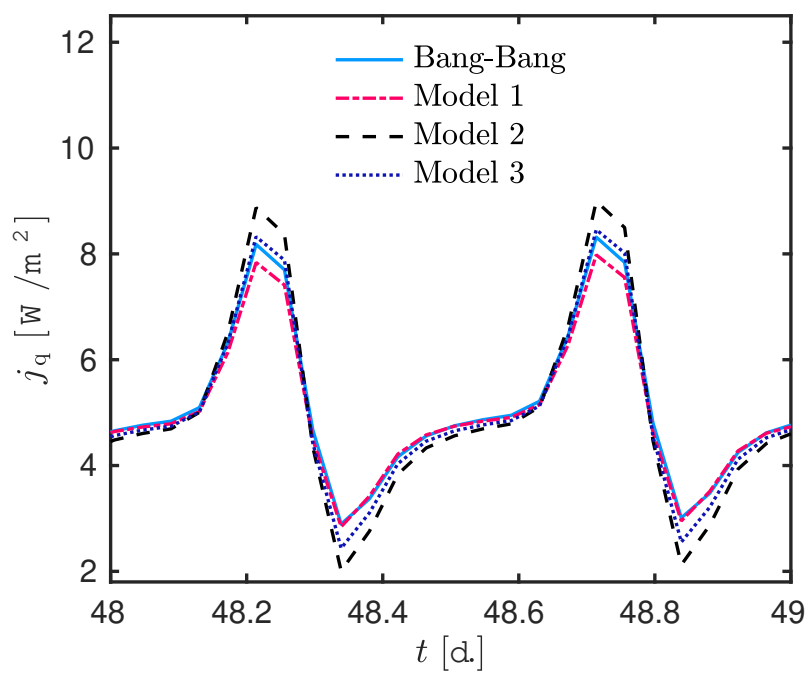

(b)

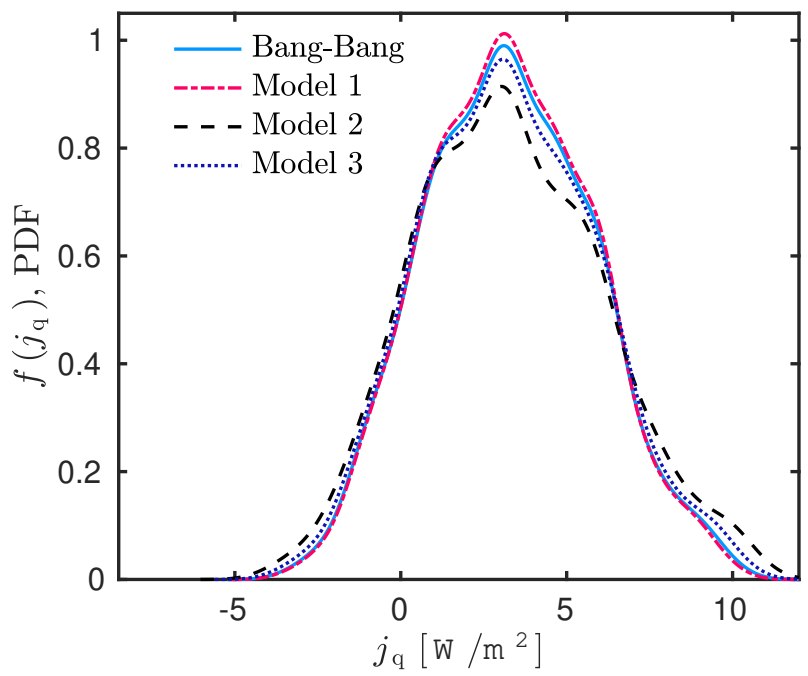

(d)

Figure 23. Time evolution of the mass flux $j_{\mathrm{m}}$ (a) and the heat flux $j_{\mathrm{q}}$ (b) at $x=L$ for the four different simulation cases. Probability density function of the mass flux $j_{\mathrm{m}}$ (c) and of the heat flux $j_{\mathrm{q}}$ (d) at $x=L$ for the four different simulation cases. 


\section{Conclusion}

It is important to build reliable and efficient numerical models to represent the physical model of heat and mass transfer in porous building material. The reliability of a model relies in its ability to represent experimental observations. In addition, the model needs to be efficient by reducing the computational efforts and providing accurate solutions. With such models, simulations can be performed to predict the physical phenomena and for instance evaluate the heat losses of a wall submitted to retrofitting.

Among the physical phenomena, the hysteresis effects on moisture sorption and moisture capacity require to be integrated in the mathematical model to ensure the reliability of the predictions $[4,7]$. Several models have been proposed in the literature, but it is still an open challenge to propose efficient numerical models for hysteresis that can be coupled with heat and moisture transport ones [10]. Thus, the objective of this paper is to explore the use of the Bang-Bang model to simulate hysteresis effects coupled with heat and mass transfer in porous materials.

Section 2 presented the mathematical model for heat and mass transfer with hysteresis effects on water content and sorption capacity. The Bang-Bang model is described. It is based on the solution of a differential equation enabling smooth transitions between the main adsorption and desorption curves. To compute the so-called intermediary scanning curves, two parameters $\beta_{\mathrm{a}}$ and $\beta_{\mathrm{d}}$ are involved in the definition of the model. These parameters control the speed of transition between one main curve to the other. In the end, the model was composed of a system of four coupled partial differential equations: two for the heat and mass balance and two for the hysteresis on the water content and sorption capacity. In Section 3, a numerical model is proposed to compute the solution with satisfactory accuracy and reduced computational cost. The heat and mass balance equations are solved using the DU FORT-FRANKEL numerical scheme. It has a strongly relaxed stability condition and an explicit definition, avoiding costly sub-iterations to treat the non-linearities. The hysteresis numerical model is based on an implicit-explicit approach. It uses the advantages of a fully implicit approach in terms of stability and consistency, but also avoids the sub-iterations at each time step due to the non-linearities. It saves important computational efforts.

The reliability of the model is investigated in Section 4 by comparing the numerical predictions with experimental data from literature [12]. Samples of hemp concrete are submitted to cycles of adsorption and desorption by increasing or decreasing the ambient relative humidity. Two cases are investigated: (i) a slow cycle of adsorption and desorption and (ii) a fast cycling case with alternating phases of adsorption and desorption. The comparison shows a very satisfactory agreement between the numerical predictions with the Bang-Bang model and the observations. The parameters $\beta_{\mathrm{a}}$ and $\beta_{\mathrm{d}}$ depend on the frequency of variation of the heat and mass transfer processes. A small increase of computational cost is gained by coupling the hysteresis model within the one of heat and mass transfer. Some investigations are required to derive an equation between the frequency of variation of the relative humidity in the material and the value of the parameters $\beta_{\mathrm{a}}$ and $\beta_{\mathrm{d}}$.

Given the reliability and efficiency of the proposed model verified, Section 5 investigated the importance of hysteresis effects on the simulation of the heat and mass transfer in a hemp concrete wall over one year. The wall is submitted to variable climate conditions at one side and daily loads of relative humidity on the inside part. An accurate prediction of the fields in the wall is obtained with the proposed mathematical model. The two parameters $\beta_{\mathrm{a}}$ and $\beta_{\mathrm{d}}$ requires to be chosen according to the frequency of variation of the fields. The importance of considering hysteresis effects in the model was highlighted by comparing the predictions obtained using either the primary adsorption, either the desorption curve, either the mean sorption curve. The predictions are more accurate when modeling hysteresis. Hysteresis effects are greater when boundary conditions are time varying and change fast. Consequently, the prediction of the heat and moisture fluxes at the indoor side is also influenced by the hysteresis phenomenon. In the end, a more reliable model is obtained for the predictions of the heat and mass transfer in building porous materials, allowing a slight increase of the computation time.

Further investigations are needed to confirm the importance of the hysteresis phenomena by comparing predictions and in-situ experimental data at the wall or building scales. 


\section{Nomenclature}

\begin{tabular}{|cll|}
\hline & \multicolumn{2}{c|}{ Latin letters } \\
$c_{\mathrm{Q}}$ & heat storage coefficient & {$\left[\mathrm{J} /\left(\mathrm{m}^{3} \cdot \mathrm{K}\right)\right]$} \\
$c_{\mathrm{M}}$ & moisture storage coefficient & {$\left[\mathrm{kg} / \mathrm{m}^{3}\right]$} \\
$c_{0}, c_{\ell}, c_{\mathrm{v}}$ & specific heat & {$[\mathrm{J} /(\mathrm{kg} \cdot \mathrm{K})]$} \\
$h_{r V}$ & surface vapour transfer coefficient & {$[(\mathrm{K} \cdot \mathrm{s}) / \mathrm{m}]$} \\
$h_{r Q}$ & surface heat transfer coefficient & {$\left[\mathrm{W} /\left(\mathrm{m}^{2} \cdot \mathrm{K}\right)\right]$} \\
$j_{m}$ & moisture flux & {$\left[\mathrm{kg} /\left(\mathrm{m}^{2} \cdot \mathrm{s}\right)\right]$} \\
$j_{q}$ & heat flux & {$\left[\mathrm{W} / \mathrm{m}^{2}\right]$} \\
$k_{\mathrm{M}}, k_{\mathrm{V}}, k_{\mathrm{L}}$ & moisture, vapor and liquid transfer coefficient & {$[\mathrm{kg} /(\mathrm{m} \cdot \mathrm{s})]$} \\
$k_{\mathrm{Q}}, k_{\mathrm{Q} 0}$ & heat transfer coefficient & {$[\mathrm{W} /(\mathrm{m} \cdot \mathrm{K})]$} \\
$k_{\mathrm{VT}}$ & vapor transfer coefficient under temperature gradient & {$[\mathrm{kg} /(\mathrm{m} \cdot \mathrm{s} \cdot \mathrm{K})]$} \\
$L$ & length & {$[\mathrm{m}]$} \\
$L_{\mathrm{v}}$ & latent heat of evaporation & {$[\mathrm{J} / \mathrm{kg}]$} \\
$P, P_{\mathrm{sat}}$ & pressure & {$[\mathrm{Pa}]$} \\
$T$ & temperature & {$[\mathrm{K}]$} \\
$t$ & time coordinate & {$[\mathrm{s}]$} \\
$x$ & space coordinate & {$[\mathrm{m}]$} \\
\hline & &
\end{tabular}

\begin{tabular}{|cll|}
\hline \multicolumn{3}{|c|}{ Greek letters } \\
$\beta$ & hysteresis coefficient & {$\left[\mathrm{s}^{-1}\right]$} \\
$\epsilon$ & emissivity & {$[\varnothing]$} \\
$\kappa_{a}$ & air permeability & {$[\mathrm{kg} /(\mathrm{m} \cdot \mathrm{s} \cdot \mathrm{Pa})]$} \\
$\mu$ & vapor permeability & {$[\varnothing]$} \\
$\Pi$ & porosity & {$[\varnothing]$} \\
$\phi$ & relative humidity & {$[\varnothing]$} \\
$\rho$ & specific mass & {$\left[\mathrm{kg} / \mathrm{m}^{3}\right]$} \\
$\omega$ & mass content & {$[\varnothing]$} \\
$\sigma$ & STEFAN-BOLTZMANN & {$\left[\mathrm{W} /\left(\mathrm{m}^{2} \cdot \mathrm{K}^{4}\right)\right]$} \\
$\xi$ & sorption capacity & {$[\varnothing]$} \\
\hline
\end{tabular}

\section{Acknowledgments}

The authors acknowledge the Junior Chair Research program "Building performance assessment, evaluation and enhancement" from the University Savoie Mont Blanc in collaboration with The French Atomic and Alternative Energy Center (CEA) and Scientific and Technical Center for Buildings (CSTB). This work was partly funded by the laboratory LOCIE thanks to the "AAP Interne".

\section{References}

[1] N. Mendes, M. Chhay, J. Berger, and D. Dutykh. Numerical methods for diffusion phenomena in building physics. PUCPress, Curitiba, Brazil, 2016. 1, 27

[2] L. A. Richards. Capillary conduction of liquids through porous mediums. Physics, 1(5):318-333, 1931. 1

[3] J. Kwiatkowski, M. Woloszyn, and J.J. Roux. Modelling of hysteresis influence on mass transfer in building materials. Building and Environment, 44(3):633-642, 2009. 1 
[4] Z. Zhang, M. Thiery, and V. Baroghel-Bouny. Numerical modelling of moisture transfers with hysteresis within cementitious materials: Verification and investigation of the effects of repeated wetting-drying boundary conditions. Cement and Concrete Research, 68:10-23, 2015. 32

[5] J. Berger, S. Gasparin, D. Dutykh, and N. Mendes. On the solution of coupled heat and moisture transport in porous material. Transport in Porous Media, 121(3):665-702, 2018. 1, 2

[6] J. Kwiatkowski, M. Woloszyn, and J.J. Roux. Influence of sorption isotherm hysteresis effect on indoor climate and energy demand for heating. Applied Thermal Engineering, 31(6):1050-1057, 2011. 1

[7] H. Derluyn, D. Derome, J. Carmeliet, E. Stora, and R. Barbarulo. Hysteretic moisture behavior of concrete: Modeling and analysis. Cement and Concrete Research, 42(10):1379-1388, 2012. 2, 32

[8] X. Zhang, W. Zillig, H.M. Künzel, C. Mitterer, and X. Zhang. Combined effects of sorption hysteresis and its temperature dependency on wood materials and building enclosures - part i: Measurements for model validation. Building and Environment, 106:143-154, 2016. 2

[9] X. Zhang, W. Zillig, H.M. Künzel, C. Mitterer, and X. Zhang. Combined effects of sorption hysteresis and its temperature dependency on wood materials and building enclosures-part ii: Hygrothermal modeling. Building and Environment, 106:181-195, 2016. 2

[10] Z. Zhang, M. Thiery, and V. Baroghel-Bouny. A review and statistical study of existing hysteresis models for cementitious materials. Cement and Concrete Research, 57:44-60, 2014. 2, 32

[11] H.J. Steeman, M. Van Belleghem, A. Janssens, and M. De Paepe. Coupled simulation of heat and moisture transport in air and porous materials for the assessment of moisture related damage. Building and Environment, 44(10):2176-2184, 2009. 2

[12] D. Lelievre, T. Colinart, and P. Glouannec. Hygrothermal behavior of bio-based building materials including hysteresis effects: Experimental and numerical analyses. Energy and Buildings, 84:617-627, 2014. $3,12,13,14,24,32$

[13] T. Colinart, D. Lelievre, and P. Glouannec. Experimental and numerical analysis of the transient hygrothermal behavior of multilayered hemp concrete wall. Energy and Buildings, 112:1-11, 2016. 2

[14] C. Rode and C.O. Clorius. Modelling of moisture transport in wood with hysteresis and temperature dependence sorption characteristics. Proceedings of the Conference Performance of Exterior Envelopes of Whole Buildings IX, Florida, 2004. 2, 24

[15] C.R. Pedersen. Prediction of moisture transfer in building constructions. Building and Environment, 27(3):387-397, 1992. 2, 24

[16] R. Remond, G. Almeida, and P. Perre. The gripped-box model: A simple and robust formulation of sorption hysteresis for lignocellulosic materials. Construction and Building Materials, 170:716-724, 2018. 2,24

[17] S. J. Dyke and B. F. Spencer. A comparison of semi-active control strategies for the mr damper. In Intelligent Information Systems, 1997. IIS '97. Proceedings, pages 580-584, 1997. 2

[18] M. Domaneschi. Simulation of controlled hysteresis by the semi-active Bouc-Wen model. Computers and Structures, 106-107:245-257, 2012.

[19] D. Burghes and A. Graham. Control and optimal control theories with applications. pages 245-266. Woodhead Publishing, 2004. 2, 5

[20] J. Berger, T. Busser, D. Dutykh, and N. Mendes. An efficient method to estimate sorption isotherm curve coefficients. Inverse Problems in Science and Engineering, 0(0):1-38, 2018. 2 
[21] I. Traoré, D. Lacroix, L. Trovalet, and G. Jeandel. Heat and moisture transport in wooden multicomposite panels. dynamic study of the air layer impact on the building envelope energetic behavior. International Journal of Thermal Sciences, 50(11):2290-2303, 2011. 2

[22] B. Remki, K. Abahri, M. Tahlaiti, and R. Belarbi. Hygrothermal transfer in wood drying under the atmospheric pressure gradient. International Journal of Thermal Sciences, 57:135 - 141, 2012. 2

[23] S. Gasparin, J. Berger, D. Dutykh, and N. Mendes. An adaptive simulation of nonlinear heat and moisture transfer as a boundary value problem. International Journal of Thermal Sciences, 133:120139, 2018. 3

[24] J. Berger, D. Dutykh, N. Mendes, and B. Rysbaiuly. A new model for simulating heat, air and moisture transport in porous building material. Submitted, pages 1-55, 2018. 8

[25] A. Nayfeh. Perturbation Methods. Wiley VCH, New York, 2000.

[26] W. Kahan and J. Palmer. On a proposed floating-point standard. ACM SIGNUM Newsletter, 14:13-21, 1979. 8

[27] E. C. Du Fort and S. P. Frankel. Stability conditions in the numerical treatment of parabolic differential equations. Mathematical Tables and Other Aids to Computation, 7(43):135-152, 1953. 9

[28] P. J. Taylor. The stability of the Dufort-Frankel method for the diffusion equation with boundary conditions involving space derivatives. The Computer Journal, 13(1):1-92, 1970. 9

[29] S. Gasparin, J. Berger, D. Dutykh, and N. Mendes. Stable explicit schemes for simulation of nonlinear moisture transfer in porous materials. Journal of Building Performance Simulation, 11(2):129-144, 2018. 9

[30] S. Gasparin, J. Berger, D. Dutykh, and N. Mendes. An improved explicit scheme for whole-building hygrothermal simulation. Building Simulation, 11(3):465-481, 2018. 9

[31] John R. Taylor. An Introduction to Error Analysis: The Study of Uncertainties in Physical Measurements. University Science Books, Sausalito, California, 1997. 13

[32] F. Collet and S. Pretot. Experimental investigation of moisture buffering capacity of sprayed hemp concrete. Construction and Building Materials, 36:58-65, 2012. 13

[33] F. Collet, J. Chamoin, S. Pretot, and C. Lanos. Comparison of the hygric behaviour of three hemp concretes. Energy and Buildings, 62:294-303, 2013.

[34] T. Colinart, P. Glouannec, and P. Chauvelon. Influence of the setting process and the formulation on the drying of hemp concrete. Construction and Building Materials, 30:372-380, 2012.

[35] T. Colinart, P. Glouannec, T. Pierre, P. Chauvelon, and A. Magueresse. Experimental study on the hygrothermal behavior of a coated sprayed hemp concrete wall. Buildings, 3(1):79-99, 2013. 13

[36] T. Pierre, T. Colinart, and P. Glouannec. Measurement of thermal properties of biosourced building materials. International Journal of Thermophysics, 35(9):1832-1852, 2014. 14

[37] A. Zaknoune, P. Glouannec, and P. Salagnac. Experimental and numerical analysis of the transient hygrothermal behavior of multilayered hemp concrete wall. Heat and Mass Transfer, 48(2):205-215, 2016. 14

[38] P.D. Hill. Kernel estimation of a distribution function. Communications in Statistics - Theory and Methods, 14(3):605-620, 1985. 15

[39] T. Busser, J. Berger, A. Piot, M. Pailha, and M. Woloszyn. Comparison of model numerical predictions of heat and moisture transfer in porous media with experimental observations at material and wall scales: an analysis of recent trends. Drying Technology, 0(0):1-33, 2018. 15 
[40] M.T. Van Genuchten. A closed-form equation for predicting the hydraulic conductivity of unsaturated soils. Soil Science Society of America, 44:892-898, 1980. 20

[41] S. Brunauer, P.H Emmett, and E. Teller. Adsorption of gases in multimolecular layers. Journal of the American Chemical Society, 60(2):309-319, 1938. 20

[42] T. Mualem and A. Beriozkin. General scaling rules of the hysteretic water retention function based on mualem's domain theory. European Journal of Soil Science, 60(4):652-661. 24

[43] A.T. Le, C. Maalouf, T. H. Mai, E. Wurtz, and F. Collet. Transient hygrothermal behaviour of a hemp concrete building envelope. Energy and buildings, 42(10):1797-1806, 2010. 24

[44] G. Costantine, C. Maalouf, T. Moussa, and G. Polidori. Experimental and numerical investigations of thermal performance of a hemp lime external building insulation. 131:140-133, 2018. 24 